\title{
Deutscher Aufsichtsrat versus Schweizer Verwaltungsrat - Systematischer Abgleich und Evaluation der zentralen Kontroll- und Leitungsgremien im deutschsprachigen Versicherungsbereich
}

\author{
Martin Eling • David Antonius Pankoke
}

Online publiziert: 15. April 2014

(C) Springer-Verlag Berlin Heidelberg 2014

Zusammenfassung Wir vergleichen die Ausgestaltung des deutschen Aufsichtsrats und des schweizerischen Verwaltungsrats und die besonderen Regelungen für Versicherer in diesen beiden Ländern. Die Regulierung beider Gremien ist ähnlich, wobei das Mandat des Verwaltungsrats grundsätzlich umfangreicher ist als das des Aufsichtsrats. Schweizer Richtlinien haben meist einen weniger bindenden Charakter als die deutschen und lassen dem Versicherungsunternehmen mehr Freiraum. Wir nehmen eine Evaluation der Regulierung anhand wissenschaftlicher Studien vor und schlussfolgern, dass die Schweizer Regulierung passgenauer und effektiver erscheint. Des Weiteren vergleichen wir die Regulierung der Aufsichtsgremien von Versicherungsunternehmen je nach Rechtsform. In beiden Ländern ist die Regulierung für börsenkotierte Aktiengesellschaften am meisten ausgeprägt, wobei die Unterschiede generell eher gering sind.

\section{German versus Swiss Supervisory Board: A systematic comparison and evaluation of central control and governing bodies in the insuran}

\begin{abstract}
We compare the supervisory boards of Swiss and German companies and the special rules for the insurers in these two countries. While the regulation is comparable, the mandate of Swiss boards is more extensive. The Swiss regulatory guidelines, however, usually have a less binding character than the German ones and let the insurance companies act more freely. We evaluate the regulation of the board regulation using results from academic studies and conclude that the Swiss regulation seems to fit better and to be more effective. In addition we compare all
\end{abstract}

D. A. Pankoke $(\varangle) \cdot$ Prof. Dr. M. Eling Institut für Versicherungswirtschaft, Universität St. Gallen, Kirchlistrasse 2, 9010 St. Gallen, Schweiz

E-Mail: david.pankoke@unisg.ch 
legal forms insurance companies can choose from regarding the regulation of the supervisory board. Although the regulation for listed share companies is highest, in both countries differences are minor.

\section{Motivation}

Ziel dieses Beitrags ist ein systematischer Abgleich der beiden zentralen Kontrollund Leitungsgremien im deutschsprachigen Versicherungsbereich: Der deutsche Aufsichtsrat versus dem schweizerischen Verwaltungsrat. Wir interessieren uns dabei sowohl für die allgemeinen gesetzlichen Anforderungen an diese Institutionen als auch für die versicherungsspezifischen Besonderheiten der Corporate Governance in Deutschland und der Schweiz. Neben dem Vergleich nehmen wir eine Evaluation der Regulierung anhand wissenschaftlicher Studien vor, um die Effektivität der gesetzgeberischen Vorgaben zu analysieren. ${ }^{1}$ Wir zeigen auch aktuelle Entwicklungen auf und leiten Implikationen für die Entwicklung der Corporate Governance im deutschsprachigen Versicherungsraum ab. Des Weiteren vergleichen wir die Regulierung der Aufsichtsgremien je nach Rechtsform.

Neuregelungen für Aufsichts- bzw. Verwaltungsräte sind derzeit in der Assekuranz von hoher Relevanz, denn zur Stärkung der Corporate Governance wurden in den vergangenen Jahren die Anforderungen an Organe der Versicherer deutlich erhöht. So haben immer mehr europäische Länder eine „Fit-and-Proper“ Anforderung auch für Mitglieder von Aufsichtsräten/Verwaltungsräten etabliert. Das heißt der Aufsichts- bzw. Verwaltungsrat muss neben allgemeinem Fachwissen das nötige strategische Versicherungswissen aufweisen, damit er seinen Aufgaben zur Beaufsichtigung und Oberleitung nachkommen kann. ${ }^{2}$ Die Rolle des Aufsichts- bzw. Verwaltungsrats wird derzeit im deutschsprachigen Raum auch unter dem Stichwort „Abzockerei“ kontrovers diskutiert. Hintergrund ist die Eidgenössische Volksinitiative ,gegen die Abzockerei“, welche Rechte vom Verwaltungsrat hin zu den Aktionären verlagert. ${ }^{3}$ Entsprechende Überlegungen werden jetzt auch für Deutschland und den gesamten EU-Raum diskutiert.

Auch die zunehmende Relevanz der neuen EU-weiten Gesellschaftsform Societas Europaea (SE) kann zur Motivation der hier betrachteten Problemstellung herangezogen werden. Vor dem Hintergrund der europäischen Integration und der zunehmenden Integration des monistischen und dualistischen Modells der Unternehmensleitung im kontinentaleuropäischen Bereich erscheint ein Abgleich der Institu-

\footnotetext{
${ }^{1}$ Bei der Beurteilung von Corporate Governance Richtlinien beschäftigen wir uns ausschließlich mit der Frage nach der Effektivität einzelner Regelungen, also der Wirksamkeit der Richtlinien auf verschiedene Unternehmensmerkmale. Effizienzgesichtspunkte, also die Frage nach der Wirksamkeit unter Berücksichtigung des Aufwands und der Kosten diskutieren wir nicht.

${ }^{2}$ Vgl. etwa Art. 12 Abs. 1 der schweizerischen Aufsichtsverordnung (AVO) oder Art. 7 Abs. 4 des deutschen Versicherungsaufsichtsgesetz (VAG).

${ }^{3}$ Zentrale Inhalte der Initiative sind ein Verbot von Abgangsentschädigungen und Vorauszahlungen für Verwaltungsräte börsenkotierter Unternehmen sowie eine jährliche Abstimmung zur Wahl aller Verwaltungsratsmitglieder und deren Vergütung. Die Initiative wurde am 3. März 2013 mit einem Ja-Stimmenanteil von $67,9 \%$ angenommen.
} 
tionen Verwaltungsrat und Aufsichtsrat relevant. ${ }^{4}$ Das monistische System mit einem gemeinsamen Board aus Executive und Non-Executive Direktoren ist insbesondere in den USA, UK und der Schweiz verbreitet. Das dualistische System mit einem separaten Aufsichtsrat und Vorstand ist dagegen das typische Leitungsmodell in vielen kontinentaleuropäischen Ländern sowie in Japan.

Neben der reinen Gegenüberstellung von Aufsichts- und Verwaltungsrat liefert dieser Beitrag einen systematischen Vergleich von Gemeinsamkeiten und Unterschieden der beiden Gremien und nimmt eine Evaluation der Effektivität der Regelungen vor. Ferner nehmen wir Verweise auf aktuelle wissenschaftliche Arbeiten zur Corporate Governance in der Assekuranz vor und betten somit die Ausarbeitung in die entsprechende wissenschaftliche Diskussion ein.

Unsere Ergebnisse zeigen Parallelen und Unterschiede zwischen dem monistisch geprägten Schweizer System und dem dualistisch geprägten deutschen System auf. So sind die Aufgaben des Schweizer Verwaltungsrats deutlich umfangreicher als die des deutschen Aufsichtsrats. Der Verwaltungsrat ist ein Leitungs- und Überwachungsorgan, während der Aufsichtsrat im Allgemeinen ein reines Überwachsungsorgan ist. Der Schweizer Verwaltungsrat ist demnach eine Institution, welche Elemente des deutschen Vorstands und Aufsichtsrats miteinander vereint. Vor dem Hintergrund der aktuellen politischen Debatte ist in den kommenden Jahren mit einer weiteren Ausweitung der Regulierung in diesem Bereich zu rechnen, in der Versicherungsbranche und darüber hinaus.

Dieser Beitrag ist wie folgt strukturiert: In Abschn. 2 stellen wir zunächst die relevanten gesetzlichen Grundlagen in Deutschland und der Schweiz gegenüber. In Abschn. 3 werden dann die allgemeinen Anforderungen an Verwaltungsräte und Aufsichtsräte in drei Blöcken abgehandelt (Organisation; Aufgaben, Haftung und Prüfung; Qualifikation und Entlohnung). Abschnitt 4 widmet sich dann den versicherungsspezifischen Anforderungen im Bereich der Corporate Governance. Abschnitt 5 vergleicht ausgewählte Anforderungen an die Aufsichtsgremien der Versicherer je nach Rechtsform. Es folgt ein Abschnitt zur Ableitung von Gemeinsamkeiten und Unterschieden sowie die Einbettung der Analyse in die wissenschaftliche Diskussion, mit dem Ziel die Effektivität der entsprechenden Regeln zu bewerten. Die Arbeit schließt mit einer Diskussion aktueller Entwicklungen und Implikationen in Abschn. 7.

\section{Gegenüberstellung der wesentlichen Grundlagen}

Corporate Governance bezeichnet den Ordnungsrahmen für die Leitung und Überwachung von Unternehmen (Vgl. Berliner Initiativkreis 2002) und ist seit vielen Jahren ein zentraler Begriff der Unternehmensführung. Wesentliche Entwicklungsschritte der Corporate Governance in Deutschland und der Schweiz sind das deutsche KonTraG (Gesetz zur Kontrolle und Transparenz im Unternehmensbereich, 1998), der Deutsche Corporate Governance Kodex (DCGK) sowie der Swiss Code of Best

\footnotetext{
${ }^{4}$ Für die SE kann grundsätzlich sowohl das dualistische als auch das monistischen Modell gewählt werden, so dass dies auch für deutsche Gesellschaften grundsätzlich in Frage kommt. Die Allianz SE und die ARAG SE haben sich zum Beispiel für ein dualistisches Modell entschieden.
} 
Tab. 1 Gegenüberstellung der wesentlichen rechtlichen Grundlagen

\begin{tabular}{|c|c|c|}
\hline Kriterium & Deutschland & Schweiz \\
\hline \multirow{2}{*}{$\begin{array}{l}\text { Corporate Governance } \\
\text { System }\end{array}$} & Dualistisch (two-tier system) & Monistisch (one-tier system) \\
\hline & $\begin{array}{l}\text { Trennung von Geschäftsführung } \\
\text { und Überwachung }\end{array}$ & $\begin{array}{l}\text { Keine Trennung von Geschäftsleitung und } \\
\text { Verwaltungsrat (Hybridform) }\end{array}$ \\
\hline \multirow{2}{*}{$\begin{array}{l}\text { Zentrale gesetzliche } \\
\text { Grundlagen }\end{array}$} & Aktiengesetz (AktG) & Bundesverfassung (BV) \\
\hline & & Obligationenrecht (OR) \\
\hline \multirow{8}{*}{$\begin{array}{l}\text { Allgemeine Cor- } \\
\text { porate Governance } \\
\text { Regelungen }\end{array}$} & KontTraG, 1998 & Swiss Code of Best Practice for Corporate \\
\hline & TransPuG, 2002 & Governance (SCBP), 2002 (Update 2007) \\
\hline & $\begin{array}{l}\text { Deutscher Corporate Gover- } \\
\text { nance Kodex (DCGK), } 2002 \\
\text { (Update } 2009,2010,2012)\end{array}$ & \\
\hline & BilReG, 2004 & SIX Swiss Exchange Richtlinie Corporate \\
\hline & VorstOG, 2005 & Governance (RLCG), 2009 \\
\hline & UMAG, 2005 & \\
\hline & BilMoG, 2009 & \\
\hline & VorstAG, 2009 & \\
\hline \multirow[t]{4}{*}{$\begin{array}{l}\text { Versicherungsspezi- } \\
\text { fische Regelungen }\end{array}$} & $\begin{array}{l}\text { Versicherungsaufsichtsgesetz } \\
\text { (VAG) }\end{array}$ & Versicherungsaufsichtsgesetz (VAG) \\
\hline & $\begin{array}{l}\text { Versicherungs-Vergütungsver- } \\
\text { ordnung (VersVergV) }\end{array}$ & Aufsichtsverordnung (AVO) \\
\hline & $\begin{array}{l}\text { Versicherungsunternehmens- } \\
\text { Rechnungslegungsverordnung } \\
\text { (RechVersV) }\end{array}$ & FINMA Rundschreiben 2008/32 \\
\hline & $\begin{array}{l}\text { Versicherungsberichterstattungs- } \\
\text { Verordnung (BerVersV) }\end{array}$ & FINMA Rundschreiben 2010/1 \\
\hline
\end{tabular}

Practice (SCBP) (jeweils eingeführt im Jahr 2002). Hinzu kommen als eine versicherungsspezifische Entwicklung spezielle Anforderungen an die Eignung und Fähigkeiten der Geschäftsleitung und des Aufsichts- bzw. Verwaltungsrats (sogenannte „Fit-and-Proper“ Anforderungen), die in anderen Industrien in der Form nicht zu finden sind. ${ }^{5}$

Zunächst erscheint es wichtig zwischen den grundsätzlich unterschiedlichen Ausprägungen des dualistischen, deutschen Systems und dem monistischen, Schweizer Systems zu trennen. Das deutsche Governance System wird als dualistisch (two-tier) System bezeichnet, d. h. es erfolgt eine Trennung von Geschäftsführung (Vorstand) und Überwachung (Aufsichtsrat). In der Schweiz ist dagegen die Geschäftsführung nicht institutionell von der Überwachung getrennt. Man spricht hier vom sogenannten monistischen System. In der Schweizer Versicherungspraxis findet sich dabei aber im Allgemeinen ein Nebeneinander von Verwaltungsrat und Geschäftsleitung (also eine Art Hybridform; Vgl. Forstmoser 2003). Der Verwaltungsrat hat dabei deutlich größere Kompetenzen und Aufgaben als der deutsche Aufsichtsrat, da er neben der Kontrolle auch die Oberleitung innehat.

Tabelle 1 zeigt eine Übersicht über die relevanten gesetzlichen Grundlagen. Für Deutschland liegen die gesetzlichen Grundlagen der Corporate Governance im

\footnotetext{
${ }^{5}$ Für weitergehende Diskussionen der Entwicklung von Corporate Governance aus Schweizer Perspektive vgl. Müller (2008) und aus deutscher Perspektive vgl. Werder (2011).
} 
Aktiengesetz (AktG). ${ }^{6}$ Bedeutende gesetzliche Initiativen mit Bezug zur Corporate Governance waren das Gesetz zur Kontrolle und Transparenz im Unternehmensbereich (KonTraG, 1998), das Gesetz zur weiteren Reform des Aktien- und Bilanzrechts, zur Transparenz und Publizität (TransPuG, 2002), das Bilanzrechtsreformgesetz (BilReG, 2004), das Vorstandsvergütungs-Offenlegungsgesetz (VorstOG, 2005), das Gesetz zur Unternehmensintegrität und Modernisierung des Anfechtungsrechts (UMAG, 2005), das Gesetz zur Modernisierung des Bilanzrechts (BilMog, 2009) sowie das Gesetz zur Angemessenheit der Vorstandsvergütung (VorstAG, 2009). ${ }^{7}$ Das Pendant zum deutschen Aktiengesetz bildet das Schweizerische Obligationenrecht (OR). Unter dem Oberbegriff ,Allgemeine Corporate Governance Regelungen “ ist insbesondere der Swiss Code of Best Practice for Corporate Governance (SCBP) zu nennen, der letztmals 2007 aktualisiert wurde. Zudem ist für börsenkotierte Gesellschaften die SIX Swiss Exchange Richtlinie Corporate Governance (RLCG), aus dem Jahr 2009 relevant, die aus dem Schweizer Börsengesetz abgeleitet ist. Seit der Annahme der oben genannten Volksinitiative lassen sich aber Corporate Governance Richtlinien auch direkt aus der Bundesverfassung (BV) ableiten.

Im Hinblick auf die versicherungsspezifischen Regelungen sind in Deutschland das Versicherungsaufsichtsgesetz (VAG), die Versicherungs-Vergütungsverordnung (VersVergV), die Verordnung für die Rechnungslegung der Versicherer (RechVersV) sowie die Versicherungsberichterstattungs-Verordnung (BerVersV) bedeutsam. Die entsprechenden Regelungen in der Schweiz nennen sich Versicherungsaufsichtsgesetz (VAG) und Aufsichtsverordnung (AVO). Zudem konkretisiert ein FINMA Rundschreiben 2008/32 Anforderungen an die Unternehmensleitung Schweizer Versicherer. Das FINMA Rundschreiben 2010/1 regelt Mindeststandards für Vergütungssysteme bei Finanzinstituten.

\section{Allgemeine Anforderungen an Verwaltungsräte und Aufsichtsräte}

Unter den allgemeinen Anforderungen verstehen wir Anforderungen, die für alle Unternehmen gelten und keinen speziellen Bezug zum Versicherungsbereich aufweisen. Diese Informationen sind insbesondere den Gesetzen und Richtlinien entnommen, die in Tab. 1 unter dem Oberbegriff „Allgemeine Corporate Governance Regelungen“ genannt werden. Wir haben die aus unserer Sicht relevanten Aspekte dabei in drei Kategorien eingeteilt: Organisation (Tab. 2), Befugnisse und Pflichten (Tab. 3) sowie Qualifikation und Entlohnung (Tab. 4).

\footnotetext{
${ }^{6}$ Wir fokussieren in diesem Beitrag die Situation insbesondere von börsenkotierten Aktiengesellschaften. Der Deutsche Corporate Governance Kodex richtet sich beispielsweise in erster Linie an börsennotierte Gesellschaften. Allerdings wird auch nicht kapitalmarktorientierten Gesellschaften, wie etwa Versicherungsvereinen, die Beachtung des Kodex empfohlen. Empirisch wurde dabei anhand von US-Daten gezeigt, dass die Leitung der Versicherungsvereine sich strukturell von Aktiengesellschaften unterscheidet. So finden sich etwa im Kontrollgremien von Versicherungsvereinen mehr unabhängige Mitglieder (d. h. Personen, die nicht mit dem Unternehmen direkt verbunden sind) als bei Aktiengesellschaften. Dadurch mag der Mangel an externer Kontrolle (keine Aktionäre, Analysten etc.) gelindert werden (Vgl. dazu Mayers et al. 1997). Ein Rechtsformvergleich mit der Herausarbeitung einiger rechtsformspezifischer Unterschiede findet sich in Abschn. 5.
}

${ }^{7}$ Vgl. Welge und Eulerich (2012), S. 3-4 und Grundei und Zaumseil (2012), S. 21-23. 
Tab. 2 Organisation

\begin{tabular}{|c|c|c|c|c|}
\hline \multirow[b]{2}{*}{ Anforderung } & \multicolumn{2}{|l|}{ Aufsichtsrat (AR) } & \multicolumn{2}{|l|}{ Verwaltungsrat (VR) } \\
\hline & Beschreibung & Quelle & Beschreibung & Quelle \\
\hline $\begin{array}{l}\text { Relevant } \\
\text { für die }\end{array}$ & Aktiengesellschaft & $\begin{array}{l}\S 30 \mathrm{AktG} ; \S 278(3) \\
\mathrm{AktG}\end{array}$ & Aktiengesellschaft & $\begin{array}{l}\text { Art. 626 E OR; } \\
\text { Art. 764(2) OR }\end{array}$ \\
\hline $\begin{array}{l}\text { Rechtsfor- } \\
\text { men }\end{array}$ & $\begin{array}{l}\text { Kommanditgesellschaft } \\
\text { auf Aktien }\end{array}$ & & $\begin{array}{l}\text { Kommanditgesell- } \\
\text { schaft }\end{array}$ & \\
\hline \multirow[t]{2}{*}{ Wahlperiode } & Maximal 5 Jahre & $\S 102 \mathrm{AktG}$ & $\begin{array}{l}1 \text { Jahr (ab März } \\
\text { 2014) }\end{array}$ & $\begin{array}{l}\text { Art. } 95 \text { Abs. } 3 \\
\text { BV; Art. } 710 \text { OR }\end{array}$ \\
\hline & & & $\begin{array}{l}\text { Maximal } 6 \text { Jahre } \\
\text { (bisher) }\end{array}$ & \\
\hline \multirow[t]{2}{*}{ Wahl } & Hauptversammlung & $\begin{array}{l}\S 30(1), \S 96 \S 101 \\
\text { AktG; DCGK } 5.4 .3\end{array}$ & Generalversammlung & Art. 698 OR \\
\hline & $\begin{array}{l}\text { Einschränkungen durch } \\
\text { Arbeitnehmermitbestim- } \\
\text { mungsgesetze }\end{array}$ & & & \\
\hline Sitzungen & $\begin{array}{l}\text { Einberufungsrecht } \\
\text { Min. } 2 \text { Sitzungen pro } \\
\text { Jahr }\end{array}$ & $\S 110(3) \mathrm{AktG}$ & Einberufungsrecht & Art. 715 OR \\
\hline \multirow[t]{2}{*}{$\begin{array}{l}\text { Mitglieder- } \\
\text { anzahl }\end{array}$} & $\begin{array}{l}3 \text { bis } 21 \text { natürliche } \\
\text { Personen }\end{array}$ & $\S 95 \mathrm{AktG}$ & $\begin{array}{l}\text { Mindestens eine } \\
\text { natürliche Person }\end{array}$ & Art. 707 OR \\
\hline & Anzahl durch 3 teilbar & & & \\
\hline \multirow[t]{2}{*}{ Diversity } & $\begin{array}{l}\text { Frauen sollen berück- } \\
\text { sichtigt werden }\end{array}$ & DCGK 5.1.2, 5.4.1 & $\begin{array}{l}\text { Internationalität } \\
\text { soll berücksichtigt } \\
\text { werden }\end{array}$ & SCBP IIb 12 \\
\hline & $\begin{array}{l}\text { Internationalität soll be- } \\
\text { rücksichtigt werden }\end{array}$ & & & \\
\hline \multirow[t]{3}{*}{ Ausschüsse } & Audit Committee & $\begin{array}{l}\text { DCGK } 5.3 .2 \text { und } \\
5.3 .3\end{array}$ & Audit Committee & $\begin{array}{l}\text { SCBP IIg 21, 23, } \\
25 \text { und } 27\end{array}$ \\
\hline & Nominierungsausschuss & & $\begin{array}{l}\text { Compensation } \\
\text { Committee }\end{array}$ & \\
\hline & & & $\begin{array}{l}\text { Nominierungsaus- } \\
\text { schuss }\end{array}$ & \\
\hline
\end{tabular}

\subsection{Organisation}

In Tab. 2 diskutieren wir die Organisation von Aufsichts- und Verwaltungsrat. Die hier beschriebenen Regeln sind relevant für Aktiengesellschaften und Kommanditgesellschaften. Wie oben bereits kurz ausgeführt sind viele der angesprochenen Aspekte auch für Genossenschaften und Versicherungsvereine relevant. Unterschiede in der Regulierung der Aufsichtsgremien je nach Rechtsform behandeln wir in Abschn. 5.

In Deutschland ist eine Benennung als Aufsichtsrat bis maximal fünf Jahre möglich, das heißt ein Aufsichtsrat kann für maximal fünf aufeinanderfolgende Hauptversammlungen gewählt werden. In der Schweiz ist dagegen bisher eine Amtsdauer von bis zu sechs Jahren möglich. Aufgrund der Annahme der Volksinitiative ,gegen die Abzockerei“" sind die Verwaltungsratsmitglieder ab März 2014 jährlich und einzeln von der Generalversammlung zu wählen. In Deutschland obliegt dies der Hauptversammlung, wobei die Mitglieder auch einzeln gewählt werden sollen. Eine Besonderheit des deutschen Aktiengesetzes, das in der Schweiz nicht bekannt ist, sind die umfangreichen Mitbestimmungsrechte der Arbeitnehmerschaft, die massive Auswir- 
Tab. 3 Befugnisse und Pflichten

\begin{tabular}{|c|c|c|c|c|}
\hline \multirow[b]{2}{*}{ Anforderung } & \multicolumn{2}{|l|}{ Aufsichtsrat (AR) } & \multicolumn{2}{|l|}{ Verwaltungsrat (VR) } \\
\hline & Beschreibung & Quelle & Beschreibung & Quelle \\
\hline \multirow[t]{4}{*}{ Aufgaben } & Kontrolle der & $\S 84$ und $\S 111$ & Oberleitung und & Art. 716a OR; \\
\hline & Gesellschaft & AktG; DCGK & & Art. 716(1) OR \\
\hline & Gewisse Entschei- & $4.1 .1,5.1 .1$ & Kontrolle der & \\
\hline & dungen des Vorstands & & Gesellschaft & \\
\hline \multirow{4}{*}{$\begin{array}{l}\text { Beschluss- } \\
\text { fassung }\end{array}$} & Mehrheitsentscheidung & \& $108 \mathrm{AktG}$ & Mehrheitsentscheidung & Art. 713 OR \\
\hline & Stimmengleichheit: Vor- & DCGK 1. & Stimmengleich- & \\
\hline & sitzender 2 Stimmen & & heit: Vorsitzender & \\
\hline & & & Stichentscheid & \\
\hline \multirow[t]{4}{*}{$\begin{array}{l}\text { Geschäfts- } \\
\text { führung }\end{array}$} & AR und Vorstand & $\S 105 \mathrm{AktG}$ & Obliegt dem VR & Art. 716(2) OR; \\
\hline & Dualistisches Svstem & 5.4 .4 & & SCBP IIe 18 \\
\hline & Dualistisches system & & $\begin{array}{l}\text { Kann aui Dritte uber- } \\
\text { tragen werden }\end{array}$ & \\
\hline & & & $\begin{array}{l}\text { Theoretisch: Monis- } \\
\text { tisches System, Prak- } \\
\text { tisch: Hybridsystem }\end{array}$ & \\
\hline \multirow[t]{3}{*}{ Haftung } & Bei Verletzung der & $\S 93$ und & Bei fahrlässiger und & Art. 754 OR \\
\hline & Sorgfaltspflicht & $\S 116 \mathrm{AktG}$ & Absichtlicher & \\
\hline & & DCGK 3.8 & Pflichtverletzung & \\
\hline $\begin{array}{l}\text { Abschluss- } \\
\text { prüfer }\end{array}$ & $\begin{array}{l}\text { Durch Hauptversamm- } \\
\text { lung bestimmt }\end{array}$ & $\begin{array}{l}\S 119 \mathrm{AktG} ; \\
\text { DCGK } 7.2 .2\end{array}$ & $\begin{array}{l}\text { Durch Generalver- } \\
\text { sammlung bestimmt }\end{array}$ & $\begin{array}{l}\text { SCBP I } 1 \text { und } \\
\text { III } 29\end{array}$ \\
\hline
\end{tabular}

kungen auf die Zusammensetzung des Aufsichtsrats haben. So ist dieser paritätisch zu besetzen. ${ }^{8}$ In der Schweiz sind entsprechende Regeln unbekannt. Der deutsche Aufsichtsrat umfasst drei bis 21 Personen, wobei die Zahl stets durch drei teilbar sein soll. In der Schweiz gibt man sich bereits mit einer natürlichen Person zufrieden. Jedes Mitglied des Aufsichtsgremiums in Deutschland wie in der Schweiz besitzt das Recht eine Sitzung einzuberufen. Der Aufsichtsrat muss mindestens zwei Mal im Kalenderjahr tagen. Im Gegensatz hierzu ist die Häufigkeit der Verwaltungsratssitzungen nicht reglementiert.

Bezüglich des Themas Diversity gibt es Vorschriften sowohl im Deutschen Corporate Governance Kodex als auch im Swiss Code of Best Practice. In beiden Regelwerken ist festgehalten, dass bei der Besetzung des Aufsichtsgremiums eine internationale Tätigkeit des Unternehmens zu berücksichtigen ist. Es sollen also nicht nur Personen aus der Heimatregion des Unternehmens im Aufsichtsgremium vertreten sein. Ein weiterer Punkt im Deutschen Corporate Governance Kodex, der die Vielfalt der vertretenden Interessensgruppen sicherstellen soll ist, dass bei der Besetzung von Vorstand und Aufsichtsrat Frauen angemessen berücksichtigt werden müssen. Regelungen bezüglich des letzteren Punktes sind im Swiss Code of Best Practice jedoch nicht enthalten.

Ein weiteres wichtiges Governance Element sind die durch den Verwaltungsrat bzw. Aufsichtsrat eingesetzten Ausschüsse. In Deutschland ist ein Audit Comitee

\footnotetext{
${ }^{8}$ Anzumerken ist, dass das Mitbestimmungsgesetz nur für große Unternehmen mit in der Regel über 2000 Mitarbeitern in vollem Umfang gültig ist, während kleinere Gesellschaften geringere Anforderungen zu erfüllen haben.
} 
Tab. 4 Qualifikation und Entlohnung

\begin{tabular}{|c|c|c|c|c|}
\hline \multirow[b]{2}{*}{ Anforderung } & \multicolumn{2}{|l|}{ Aufsichtsrat (AR) } & \multicolumn{2}{|l|}{ Verwaltungsrat (VR) } \\
\hline & Beschreibung & Quelle & Beschreibung & Quelle \\
\hline \multirow[t]{4}{*}{ Qualifikation } & $\begin{array}{l}\text { Beschränkte Anzahl } \\
\text { Mandate }\end{array}$ & $\begin{array}{l}\S 100(2) \mathrm{AktG} \\
\text { DCGK 5.4.1 }\end{array}$ & $\begin{array}{l}\text { Laut Gesetzt: keine } \\
\text { spezielle Qualifika- } \\
\text { tion erforderlich }\end{array}$ & $\begin{array}{l}\text { Art. } 718 \text { OR; Art. } \\
716 a(1) 3 \text { OR; } \\
\text { SCBP IIb } 12\end{array}$ \\
\hline & Keine Kreuzverflechtung & & Eine Person wohn- & \\
\hline & $\begin{array}{l}\text { Kein Vorstand der letzten } \\
2 \text { Jahre }\end{array}$ & & haft in der Schweiz & \\
\hline & $\begin{array}{l}\text { AR soll erforderlichen } \\
\text { Kenntnisse, Fähigkeiten und } \\
\text { fachliche Erfahrung besitzen }\end{array}$ & & & \\
\hline \multirow{5}{*}{$\begin{array}{l}\text { Entlohnung } \\
\text { Vorstand/ } \\
\text { Geschäfts- } \\
\text { führung }\end{array}$} & Entscheidung AR & $\begin{array}{l}\S 87 \mathrm{AktG} \\
\text { DCGK } 4.2 .2 \\
4.2 .3,4.2 .4\end{array}$ & $\begin{array}{l}\text { Entscheidung Gene- } \\
\text { ralversammlung (ab } \\
\text { März 2014) }\end{array}$ & $\begin{array}{l}\text { Art. } 95 \text { Abs. } 3 \\
\text { BV; Art. } 663 b^{\text {bis }} \\
\text { OR; SCBP An- }\end{array}$ \\
\hline & Fixe und variable Anteile & und 4.2 .5 & $\begin{array}{l}\text { Verantwortung VR } \\
\text { (bisher) }\end{array}$ & $\begin{array}{l}\text { hang a } 1, \text { b } 3,4,6 \text {, } \\
7, \text { c } 8,9\end{array}$ \\
\hline & $\begin{array}{l}\text { Abfindung beim Ausschei- } \\
\text { den soll maximal } 3 \text { Jahres- } \\
\text { gehälter betragen }\end{array}$ & & $\begin{array}{l}\text { Fixe und variable } \\
\text { Anteile }\end{array}$ & \\
\hline & Individuelle Offenlegung & & $\begin{array}{l}\text { Es soll/darf keine } \\
\text { Abgangs-entschädi- } \\
\text { gungen geben (bis- } \\
\text { her/ab März 2014) }\end{array}$ & \\
\hline & & & $\begin{array}{l}\text { Kollektive } \\
\text { Offenlegung }\end{array}$ & \\
\hline \multirow{6}{*}{$\begin{array}{l}\text { Entlohnung } \\
\text { VR/AR }\end{array}$} & Entscheidung & $\S 113 \mathrm{AktG}$ & Ab März 2014: & Art. 95 Abs. 3 BV \\
\hline & Hauptversammlung & DCGK 5.4.6 & & Art. $663 b^{\text {bis }}$ OR; \\
\hline & $\begin{array}{l}\text { Fixe und variable } \\
\text { Bestandteile }\end{array}$ & & $\begin{array}{l}\text { Entscheidung } \\
\text { Generalversammlung } \\
\text { Bisher: }\end{array}$ & $\begin{array}{l}\text { SCBP Anhang a } \\
1, \mathrm{~b} 4,6,7, \mathrm{c} 8,9\end{array}$ \\
\hline & Individuelle Offenlegung & & Entscheidung VR & \\
\hline & & & $\begin{array}{l}\text { Branche } \\
\text { Vergleichsmaßstab }\end{array}$ & \\
\hline & & & $\begin{array}{l}\text { Individuelle } \\
\text { Offenlegung }\end{array}$ & \\
\hline
\end{tabular}

vorgesehen, dass nicht vom Aufsichtsratsvorsitzenden geleitet werden soll und ein Nominierungsausschuss. Ausschüsse hingegen, die Vorstandsverträge behandeln sollen durch den Aufsichtsratsvorsitzenden geleitet werden. Die Schweiz sieht mit dem Audit Committee, dem Compensation Committee und dem Nominierungsausschuss bereits die Bildung von drei Institutionen vor, wobei sowohl das Audit als auch das Compensation Commitee hauptsächlich bzw. wenn möglich ausschließlich aus unabhängigen Verwaltungsräten bestehen soll.

Für eine weitergehende Diskussion der Organisation des Aufsichtsrats siehe Roth (2012) und des Verwaltungsrats siehe Bauen und Venturi (2007), S. 83-151.

\subsection{Befugnisse und Pflichten}

Die Aufgaben des deutschen Aufsichtsrats sind im $\S 111$ AktG und DCGK 4.1.1, 5.1.1 festgelegt (Vgl. Tab. 3). Im Kern geht es um die Kontrolle der Gesellschaft, wobei 
die Einbindung des Aufsichtsrats bei strategischen Entscheidungen vorgesehen ist. Gewisse Entscheidungen des Vorstands sind dabei zustimmungspflichtig, sofern dies die Satzung der Gesellschaft vorsieht (häufig z. B. bei Kapitalerhöhungen). Außerdem hat der Aufsichtsrat für den Vorstand eine Beratungsfunktion. Der Schweizer Verwaltungsrat hat sowohl die Oberleitung als auch die Kontrolle der Gesellschaft zu übernehmen (Vgl. Art. 716a OR; Art. 716(1) OR). Ferner ist er beschlussfähig zu allen Angelegenheiten, die nicht durch Gesetz oder Statuten der Generalversammlung vorbehalten sind.

Die Aufgabe des Aufsichtsrats und des Verwaltungsrats ist die Kontrolle der Gesellschaft. Zusätzlich ist der Verwaltungsrat für die Oberleitung des Unternehmens verantwortlich. Im Allgemeinen wird der Aufsichtsrat nur bei strategischen Entscheidungen eingebunden; er kontrolliert, nominiert und berät den Vorstand. ${ }^{9}$ Gewisse Entscheidungen des Vorstands sind zudem zustimmungspflichtig. Der Verwaltungsrat hingegen ist zu allen Angelegenheiten, die nicht durch Gesetz oder Statuten der Generalversammlung vorbehalten sind beschlussfähig. In beiden Gremien werden Beschlüsse durch eine Mehrheitsentscheidung herbeigeführt, wobei der Vorsitzende bei Stimmengleichheit den Ausschlag gibt.

Wie oben erwähnt herrscht in Deutschland ein dualistisches System. Aufsichtsratsmitglieder dürfen nicht der Geschäftsführung angehören. Auch sollen dem Aufsichtsrat nicht mehr als zwei ehemalige Vorstände angehören und eine zweijährige Karenzzeit vor einem Wechsel vom Vorstand in den Aufsichtsrat soll eingehalten werden. Bei letzterem sind Ausnahmen jedoch möglich. In der Schweiz hingegen herrscht ein monistisches System und die Geschäftsführung obliegt dem Verwaltungsrat. Diese kann jedoch auf einzelne Mitglieder oder Dritte übertragen werden. Der Verwaltungsrat entscheidet zudem ob die Geschäftsführung und der Verwaltungsrat von der gleichen Person geleitet werden. Da dies in der Regel der Fall ist spricht man von dem Schweizer Modell auch als Hybridsystem.

Mitglieder des Aufsichtsgremiums können bei einer Verletzung der Sorgfaltspflicht (Aufsichtsrat) oder bei einer fahrlässigen und absichtlichen Pflichtverletzung (Verwaltungsrat) haftbar gemacht werden. ${ }^{10}$ In Deutschland sollen zudem Directors and Officers Versicherungen welche die finanziellen Konsequenzen dieses Fehlverhaltens abdecken einen Selbstbehalt enthalten. Der Abschlussprüfer wird jeweils durch die Hauptversammlung/Generalversammlung bestimmt und soll in Deutschland durch den Aufsichtsrat beauftragt werden.

\subsection{Qualifikation und Entlohnung}

Zwei zentrale Elemente der Corporate Governance sind die Fragen der Qualifikation und Entlohnung (Vgl. Tab. 4). Neuere Entwicklungen der Corporate Governance haben dazu geführt, dass im Allgemeinen eine Obergrenze für die Anzahl der Aufsichtsrats- bzw. Verwaltungsratsmandate pro Mitglied für sinnvoll erachtet wird, um

\footnotetext{
${ }^{9}$ Vgl. auch mit Prigge (2012) und Grün und Schmitz (2012).

${ }^{10}$ Für weitergehende Diskussionen der Haftung von Aufsichtsratsmitgliedern siehe Zaumseil (2012) und Van Betteray und Per Heerma (2011). In Bezug auf die Haftung von Verwaltungsratsmitgliedern siehe Bauen und Venturi (2007) S. 261-291 und Müller et al. (2007), S. 308-315.
} 
den damit verbundenen Aufgaben verantwortungsvoll nachkommen zu können. So fordert das deutsche Aufsichtsrecht, dass eine Person nicht mehr als zehn Aufsichtsratsmandate innehaben darf (drei falls parallel Vorstandstätigkeit). Auch soll der Aufsichtsrat nicht Vorstand der Gesellschaft innerhalb der letzten zwei Jahre gewesen sein. Hintergrund ist, dass der Aufsichtsrat nicht die Beurteilung der in den Vorjahren selbst getroffenen Entscheidungen vornehmen soll. Des Weiteren darf dem Aufsichtsrat der Gesellschaft kein gesetzlicher Vertreter eines abhängigen Unternehmens angehören oder ein gesetzlicher Vertreter einer Kapitalgesellschaft, dessen Aufsichtsrat Vorstand der Gesellschaft ist. ${ }^{11}$ Im Falle des Verwaltungsrats ist vom Gesetz her keine spezielle Qualifikation erforderlich, außer dass mindestens eine Person, welche befugt ist die Gesellschaft nach außen zu vertreten in der Schweiz wohnhaft sein muss. Indirekt werden Qualifikationen jedoch vorausgesetzt für Rechnungswesen, Finanzkontrolle und Finanzplanung. ${ }^{12}$ Auch sollen VR-Mitglieder nach dem SCBP die erforderlichen Fähigkeiten besitzen, „....damit eine eigenständige Willensbildung im kritischen Gedankenaustausch mit der Geschäftsleitung gewährleistet ist.“

Die Entlohnung des Vorstands wird in Deutschland und momentan auch noch in der Schweiz vom Aufsichtsgremium festgesetzt. Ab März 2014 bestimmt jedoch die Generalversammlung in der Schweiz die Entlohnung der Geschäftsführung. Die Entlohnung des Vorstands soll sich in beiden Ländern aus einem fixen und einem variablen Anteil zusammensetzen. In Deutschland soll der Aufsichtsrat zudem beachten, dass der variable Vergütungsanteil eine mehrjährige Bemessungsgrundlage besitzt und bezüglich außerordentlicher Geschäftsentwicklungen begrenzt ist. Eine Abfindung beim Ausscheiden des Vorstandsmitglieds soll maximal drei Jahresgehälter betragen. ${ }^{13}$ In der Schweiz soll der Verwaltungsrat mittel- und langfristig ausgerichtete Anreize bei der Vergütung der Geschäftsführung setzen und sich bei der Festsetzung der Entlohnung an anderen Unternehmen orientieren. Eine Abgangsentschädigung für die Geschäftsführung soll es momentan nicht geben und ist ab März 2014 verboten.

Eine wichtige Rolle spielt nicht nur die Höhe der Entlohnung, sondern auch die Offenlegung der Vergütung der Vorstände. In Deutschland soll die Vergütung eines jeden Vorstands im Corporate Governance Bericht in einem Vergütungsteil erfolgen. In der Schweiz sollen die Bezüge der Geschäftsführung als Ganzes und des höchstbezahlten Mitglieds offen gelegt werden. Hierzu wird die Generalversammlung in einem Entschädigungsbericht über das Entschädigungssystem informiert.

Die Entlohnung für das Aufsichtsgremium selbst wird in Deutschland von der Hauptversammlung festgelegt und soll fixe sowie variable Bestandteile enthalten. Im Corporate Governance Bericht soll die Vergütung des Aufsichtsrats individualisiert öffentlich gemacht werden. In der Schweiz wird die Entlohnung ab März 2014 für den Verwaltungsrat von der Generalversammlung festgelegt und Abgangsvergütungen, Vergütungen im Voraus oder Prämien für M\&A-Aktivitäten werden verboten. Momentan wird die Vergütung des Verwaltungsrats jedoch noch vom Entschädi-

\footnotetext{
${ }^{11}$ Siehe auch Leube (2012) für eine weitergehende Diskussion.

${ }^{12}$ Für eine weitergehende Diskussion bzgl. der erforderlichen Qualifikation von Verwaltungsratsmitgliedern siehe auch Bauen und Venturi (2007), S. 5-11.

${ }^{13}$ Für eine weitergehende Diskussion der Vergütung von Aufsichtsräten siehe Hönsch und Kaspar (2012).
} 
gungsausschuss des Verwaltungsrats selbst festgelegt. Die Entlohnung in anderen Unternehmen soll hierbei als Vergleichsmaßstab dienen. Eine Offenlegung der Verwaltungsrats Entlohnung soll auf individueller Ebene im Entschädigungsbericht über das Entschädigungssystem an die Generalversammlung erfolgen.

\section{Versicherungsspezifische Anforderungen im Versicherungsbereich}

Unter spezielle Anforderungen diskutieren wir diejenigen Anforderungen, welche sich speziell an Gremien von Versicherungsunternehmen richten. Diese Informationen sind insbesondere den Gesetzen und Richtlinien entnommen, die in Tab. 1 unter dem Oberbegriff „Versicherungsspezifische Regelungen“ genannt werden. Ein Beispiel für eine solche Anforderung ist, dass in der Schweizer Assekuranz eine Personaleinheit von Vorsitz des Verwaltungsrat und der Geschäftsführung nicht gestattet ist. Dies stellt eine Abweichung vom allgemeinen Grundsatz dar, die eine solche Personaleinheit grundsätzlich ermöglicht.

Es gibt viele Besonderheiten der Versicherungsindustrie bezüglich der Ausgestaltung von Corporate Governance (Vgl. Tab. 5). Hintergrund ist die besondere volkswirtschaftliche Funktion und damit einhergehend die staatliche Aufsicht über Versicherungsunternehmen. Diese dient dem Schutz der Versicherungsnehmer sowie der Sicherstellung der Funktionsfähigkeit des Versicherungsmarkts. Aufsichtsrechtliche Eingriffe können dabei so weit gehen, dass Aufsichts- bzw. Verwaltungsratsmitglieder abberufen werden können. ${ }^{14}$

Eine wichtige Besonderheit stellt in diesem Zusammenhang auch die „Fit-andProper“ Anforderung dar. Demnach müssen Aufsichtsräte und Verwaltungsräte das notwendige Fachwissen sowie die persönliche Eignung zur Durchführung der Aufgabe mitbringen. Das notwendige Fachwissen kann beispielsweise aus einer Führungsfunktion im Versicherungsbereich erworben werden. Im deutschen System wird die persönliche Eignung dahingehend spezifiziert, dass die betreffende Person zuverlässig sein muss und die entsprechende Sachkunde besitzt. Im Übrigen dürfen höchsten zwei ehemalige Geschäftsleiter im Aufsichtsrat vertreten sein. Im Schweizer System wird die Eignung über Fachwissen, Erfahrung und guten Ruf definiert. Das Fachwissen wird weitergehend spezifiziert als strategisches Versicherungswissen, insbesondere um das Geschäft, die Prozesse und Risiken des Unternehmens verstehen und beurteilen zu können. Ist die Qualifikation nicht ausreichend oder wird den Gesetzen und Verordnungen nicht Folge geleistet, so können die deutschen sowie die Schweizer Aufsichtsbehörden den oder die betreffenden Personen aus dem Aufsichts- bzw. Verwaltungsrat abberufen. Zudem kann die betreffende Person mit einem Berufsverbot von bis zu fünf Jahren belegt werden (Vgl. Bähr et al. 2012, S. 579580). Bedeutsam erscheinen hier auch die Anforderungen, die aus dem deutschen MA Risk (Mindestanforderungen an das Risikomanagement von Versicherern) kom-

\footnotetext{
${ }^{14}$ Neben der staatlichen Aufsicht und damit einhergehenden Anforderungen gibt es noch weitere spezifische Besonderheiten im Bereich der Aufsicht und Kontrolle, so etwa dass Rückversicherer und Intermediäre (Broker, Agenten), die Qualität von Versicherern analysieren. Aufgrund der ex-post Finanzierung von Garantieeinrichtungen haben Versicherer mitunter sogar einen Anreiz sich gegenseitig zu kontrollieren (Vgl. Downs und Sommer 1999).
} 
Tab. 5 Spezielle Anforderungen im Versicherungsbereich

\begin{tabular}{|c|c|c|c|c|}
\hline \multirow[b]{2}{*}{ Anforderung } & \multicolumn{2}{|l|}{ Aufsichtsrat (AR) } & \multicolumn{2}{|l|}{ Verwaltungsrat (VR) } \\
\hline & Beschreibung & Quelle & Beschreibung & Quelle \\
\hline \multirow[t]{3}{*}{ Qualifikation } & $\begin{array}{l}\text { Fit-and-Proper } \\
\text { (Zuverlässigkeit, } \\
\text { Fachwissen) }\end{array}$ & $\begin{array}{l}\S 7 \mathrm{a}(4) \text { und } \\
\S 87(8) \text { VAG }\end{array}$ & $\begin{array}{l}\text { Fit-and-Proper (notwen- } \\
\text { diges Fachwissen, Erfah- } \\
\text { rung und guten Ruf) }\end{array}$ & \multirow{3}{*}{$\begin{array}{l}\text { Art. 14, 22, 51(1,2f) } \\
\text { und } 67 \text { VAG; Art. } \\
12 \text { und } 14 \text { AVO; } \\
\text { FINMA Rundschrei- } \\
\text { ben } 2008 / 32 \text { III.(B.) }\end{array}$} \\
\hline & $\begin{array}{l}\text { Max. } 2 \\
\text { ex-Geschäftsleiter }\end{array}$ & & $\begin{array}{l}\text { Versicherungswissen im } \\
\text { VR vorhanden }\end{array}$ & \\
\hline & $\begin{array}{l}\text { Abberufung/ } \\
\text { Berufsverbot } \\
\text { möglich }\end{array}$ & & $\begin{array}{l}\text { Abberufung/Berufsver- } \\
\text { bot möglich }\end{array}$ & \\
\hline \multirow[t]{4}{*}{$\begin{array}{l}\text { Mögliche } \\
\text { Rechtsform }\end{array}$} & $\begin{array}{l}\text { Aktiengesell- } \\
\text { schaft (AG) }\end{array}$ & \multirow{4}{*}{$\begin{array}{l}\S 7(1) \mathrm{VAG} ; \\
\S 30(1) \mathrm{AktG} \\
\S 35 \mathrm{VAG}\end{array}$} & $\begin{array}{l}\text { Aktiengesellschaft mit } \\
\text { VR }\end{array}$ & \multirow{4}{*}{$\begin{array}{l}\text { Art. } 7 \text { VAG; Art. } 698 \\
\text { OR; Art. 894(1) OR; } \\
\text { Art. 902(2) OR }\end{array}$} \\
\hline & $\begin{array}{l}\text { Societas Euro- } \\
\text { paea }(\mathrm{SE})\end{array}$ & & \multirow{3}{*}{$\begin{array}{l}\text { Genossenschaft mit } \\
\text { VR(Aufgaben: Ober- } \\
\text { leitung/Kontrolle } \\
\text { Geschäftsführung) }\end{array}$} & \\
\hline & $\begin{array}{l}\text { Körperschaft } \\
\text { des öffentlichen } \\
\text { Rechts sein } \\
\text { (KdöR) }\end{array}$ & & & \\
\hline & $\begin{array}{l}\text { Versicherungs- } \\
\text { verein auf Gegen- } \\
\text { seitigkeit (VVaG) }\end{array}$ & & & \\
\hline $\begin{array}{l}\text { Doppel- } \\
\text { funktion }\end{array}$ & $\begin{array}{l}\text { Nicht möglich } \\
\text { (wie oben) }\end{array}$ & $\S 105 \mathrm{AktG}$ & $\begin{array}{l}\text { Nicht möglich beim } \\
\text { Vorsitzenden }\end{array}$ & Art. 13 AVO \\
\hline $\begin{array}{l}\text { Interessen- } \\
\text { konflikte }\end{array}$ & $\begin{array}{l}\text { Interessenkonflik- } \\
\text { te sind offen } \mathrm{zu} \\
\text { legen }\end{array}$ & DCGK 5.5.2 & $\begin{array}{l}\text { Interessenkonflikte sind } \\
\text { zu vermeiden }\end{array}$ & $\begin{array}{l}\text { Art. III B(5) FINMA } \\
\text { Rundschreiben } \\
\text { 2008/32 III.(B.); } \\
\text { SCBP IId } 16\end{array}$ \\
\hline Vergütung & $\begin{array}{l}\text { Hauptversamm- } \\
\text { lung }\end{array}$ & $\begin{array}{l}\text { Versicherungs- } \\
\text { Vergütungsverord- } \\
\text { nung (VersVergV) }\end{array}$ & $\begin{array}{l}\text { VR erlässt Vergütungs- } \\
\text { reglement, welches alle } \\
\text { Mitarbeiter umfasst }\end{array}$ & $\begin{array}{l}\text { FINMA Rundschrei- } \\
\text { ben } 2010 / 1\end{array}$ \\
\hline
\end{tabular}

men, welche verbindliche Vorgaben für die Ausgestaltung des Risikomanagements in deutschen Versicherungsunternehmen darstellen. Gemäß dem Proper Kriterium unterliegen Personen, die in der Geschäftsleitung sind, strengeren Missbrauchsvorschriften im Vergleich zu nicht beaufsichtigten Marktteilnehmern. ${ }^{15}$ In der Schweizer Verordnung über die Aufsicht von privaten Versicherungsunternehmen (AVO) wird zudem explizit nach vorhandenem Versicherungswissen im Verwaltungsrat verlangt.

Interessenkonflikte werden in Deutschland sowie in der Schweiz in den allgemeinen Corporate Governance Richtlinien behandelt. In der Schweiz werden zudem explizit Versicherungsunternehmen in dem FINMA Rundschreiben 2008/32 darauf hingewiesen, dass Mitglieder des Verwaltungsrats persönliche Interessenkonflikte zu vermeiden haben.

In Bezug auf das Vergütungssystem speziell von Banken und Versicherungen ist in Deutschland die Versicherungsvergütungsverordnung (VersVegV) und in der Schweiz das FINMA Rundschreiben 2010/1 relevant. Beide Verordnungen sind darauf fokussiert das Vergütungssystem für den Vorstand bzw. für die Geschäftsleitung

\footnotetext{
${ }^{15}$ Für eine weitergehende Diskussion des „Fit-and-Proper“ Kriteriums im Rahmen von Solvency II siehe Pohlmann (2012).
} 


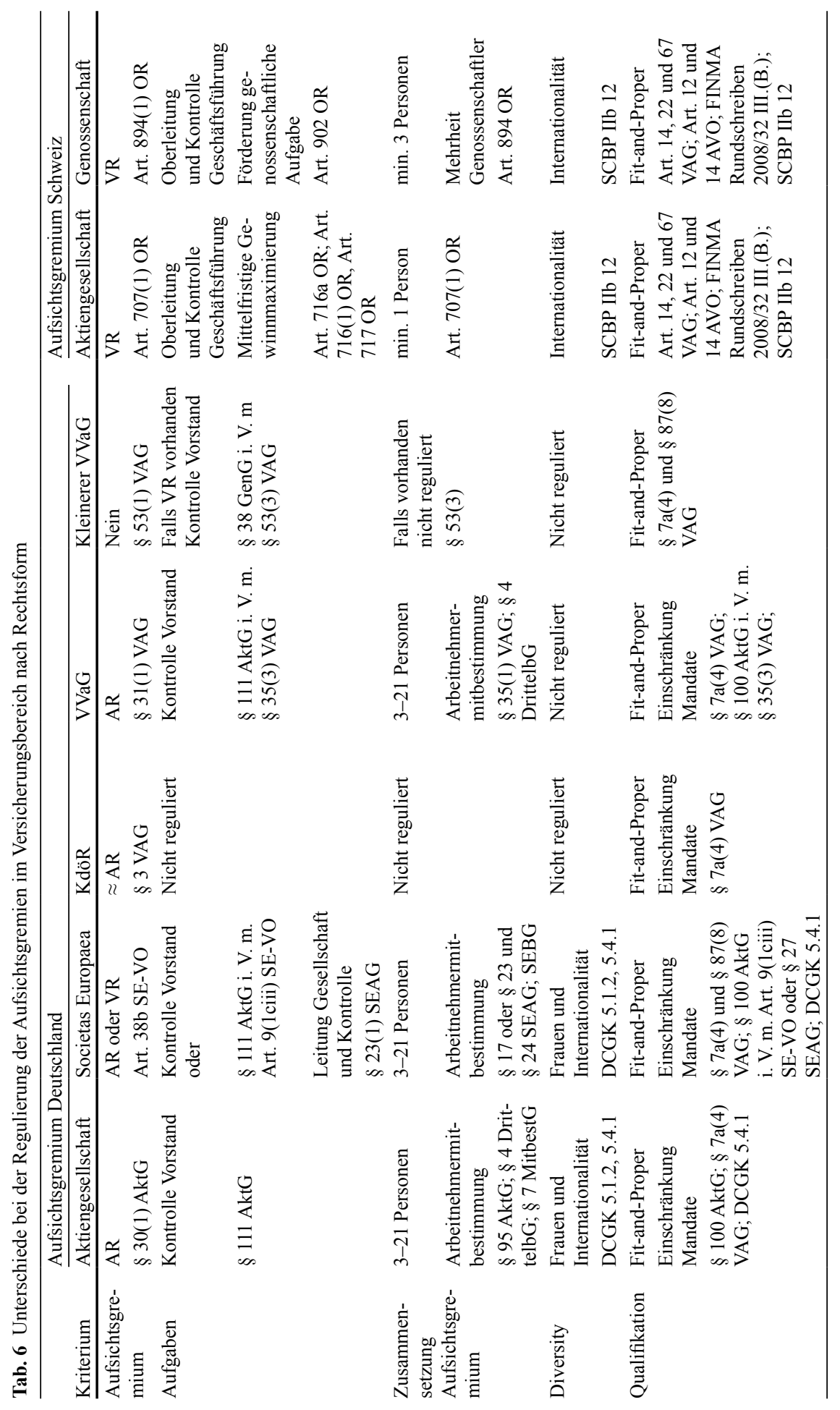


৩

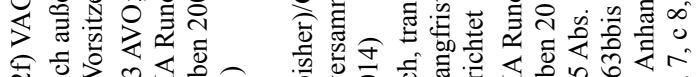

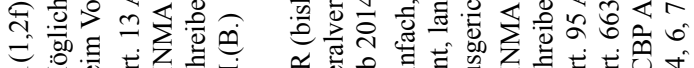

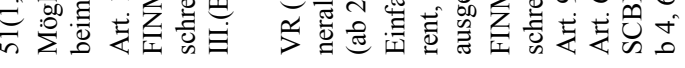

\section{$\Xi$}

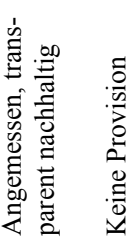

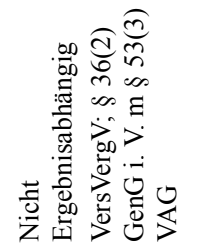

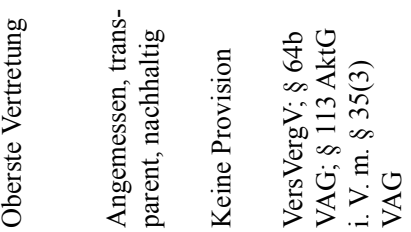

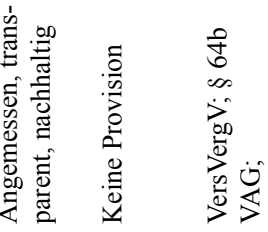

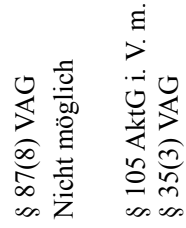

0.
$\infty$
$\infty$
$\infty$
$\infty$
$\infty$
$\infty$
$\infty$

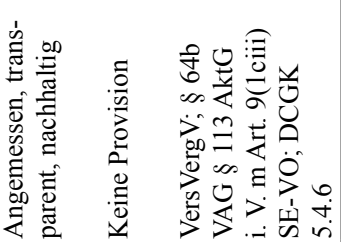


zu regulieren. Es wird darauf hingewiesen, dass hierfür jeweils der Aufsichts- bzw. Verwaltungsrat verantwortlich ist. Ferner darf die Vergütung für deutsche Aufsichtsräte nicht im Zusammenhang mit der Vermittlung von Versicherungsverträgen stehen.

\section{Rechtsformvergleich}

In Bezug auf die Rechtsform muss es sich bei einer deutschen Versicherung um eine Aktiengesellschaft, Societas Europaea, einem Versicherungsverein auf Gegenseitigkeit oder einer Körperschaft des öffentlichen Rechts handeln. In der Schweiz hingegen kann eine Versicherung entweder eine Aktiengesellschaft oder Genossenschaft sein. Tabelle 6 stellt die Unterschiede in der Ausgestaltung der Aufsichtsgremien nach Rechtsform gegenüber.

In Deutschland müssen Versicherungen in Form einer Aktiengesellschaft oder eines Vereins auf Gegenseitigkeit prinzipiell einen Aufsichtsrat bilden. Eine Körperschaft des öffentlichen Rechts (KdöR) muss ein äquivalentes Aufsichtsorgan besitzen. Ausnahmen sind die Societas Europae (SE) und der kleinere Versicherungsverein auf Gegenseitigkeit (kleinere VVaG). Bei ersterer Rechtsform ist bei Gründung der Gesellschaft zu entscheiden, ob das monistische System mit einem Verwaltungsrat eingeführt werden soll oder ein dualistisches mit Vorstand und Aufsichtsrat. VVaGs, welche örtlich begrenzt Versicherungen ausschließlich an Mitglieder vertreiben und von den Aufsichtsbehörden als ,kleinerer Verein“ eingeschätzt werden, sind permanent davon befreit einen Aufsichtsrat bilden zu müssen. In der Schweiz muss für eine Aktiengesellschaft sowie für eine Genossenschaft zwingend ein Verwaltungsrat benannt werden.

Die Hauptaufgabe des Aufsichtsgremiums liegt bei der AG, der SE mit Aufsichtsrat und dem VVaG auf der Kontrolle des Vorstands. Nur im Falle der SE mit Verwaltungsrat kommt dem Verwaltungsrat zusätzlich eine Leitungsfunktion zu. Dies ähnelt dem System in der Schweiz wo der Verwaltungsrat grundsätzlich die Oberleitung und Kontrolle der Geschäftsführung innehat. Ein gewichtiger Unterschied zwischen den Aufgaben der AG und der Genossenschaft in der Schweiz besteht jedoch darin, dass nur der Verwaltungsrat der AG dem Ziel der mittelfristigen Gewinnmaximierung zu folgen hat (Vgl. Honsell et al. 2008, S. 1094). Der Verwaltungsrat einer Genossenschaft hingegen hat primär die Pflicht, die genossenschaftliche Aufgabe zu fördern (Vgl. Honsell et al. 2008, S. 2050). Die KdöR in Deutschland ist bzgl. ihrer Aufgaben nicht reguliert.

Auch bzgl. der Zusammensetzung des Aufsichtsgremiums ist die KdöR nicht reguliert. Das gleiche gilt für den kleineren VVaG. Die Aufsichtsgremien der anderen möglichen Rechtsformen in Deutschland müssen aus einer Anzahl von zwischen drei und 21 Personen bestehen. Auch die Arbeitnehmermittbestimmung ist bei allen drei Rechtsformen garantiert. Interessant ist besonders die SE, da hier das Mitbestimmungsniveau frei zwischen Arbeitgebern und Arbeitnehmern verhandelt werden kann. ${ }^{16}$ Können sich die beiden Parteien nicht einigen, so sind die Bestimmungen

\footnotetext{
${ }^{16}$ Besonders dieser Freiraum, dass die Arbeitnehmermitbestimmung neu vereinbart und das Aufsichtsgremium verkleinert werden kann können Motive für die Umwandlung einer AG in eine SE sein (Vgl.
} 
maßgebend, die vor der Umwandlung in die SE galten (Vgl. Ziegler und Gey 2009). Wie bereits erwähnt kennt das Schweizer Gesetz keine Arbeitnehmermitbestimmung. Der Verwaltungsrat einer AG kann jedoch aus einer Person bestehen, wobei die Genossenschaft mindestens drei Personen erfordert. Mehr als die Hälfte davon müssen Genossenschaftler sein.

Fit-and-Proper Anforderungen bzgl. der Qualifikation der Mitglieder des Aufsichtsgremiums gibt es in Deutschland und der Schweiz für alle Rechtsformen. Wie oben erwähnt, ist eine Abberufung der Mitglieder des Aufsichtsgremiums sowie die Verhängung von Berufsverboten durch die Aufsichtsbehörden prinzipiell in allen Rechtsformen möglich.

Unterschiede ergeben sich hauptsächlich durch weitergehende Regelungen im DCGK für Deutschland, welcher sich primär an börsenkotierte Aktiengesellschaften richtet. ${ }^{17}$ So gibt es z. B. keine Vorgaben inwieweit Frauen oder Personen mit verschiedenen kulturellen Hintergründen in Aufsichtsgremien von KdöRs oder VVaGs vertreten sein sollen. In der Schweiz bezieht sich der SCBP auch hauptsächlich auf börsenkotierte Unternehmen, kann jedoch als bloße Empfehlung ausdrücklich auch auf andere Unternehmen ausgedehnt werden. In der Schweiz sehen wir also keine Unterschiede bezogen auf die Rechtsform, welche aus dem SCBP resultieren würden.

In der Schweiz dürfen einfache Mitglieder des Verwaltungsrats Doppelfunktionen einnehmen, d. h. sowohl in der Geschäftsführung als auch im Aufsichtsgremium mitwirken. Vorsitzende der Organe dürfen laut Versicherungsaufsichtsgesetz keine Doppelfunktionen innehaben. Ausnahmen sind jedoch mit Erlaubnis der FINMA möglich. In Deutschland ist bei den meisten Rechtsformen das Ausüben von Doppelfunktionen untersagt. Ausnahmen bilden die SE, falls das monistische System gewählt wurde und die KdöR, welche in dieser Hinsicht nicht durch das Gesetz reguliert ist.

Die Entlohnung des Aufsichtsgremiums in der Versicherungsbranche in Deutschland folgt ähnlichen Regelungen über alle Rechtsformen. Die Hauptversammlung bei AGs bzw. die oberste Vertretung bei VVaGs entscheidet über die Höhe der Vergütung. Eine Ausnahme bildet die KdöR, welche per Gesetz in dieser Hinsicht nicht reguliert ist. Für alle Rechtsformen in Deutschland gilt jedoch, dass die Vergütung angemessen, transparent und nachhaltig zu gestalten ist (Vgl. Bähr et al. 2012, S. 443-444). Zudem darf keine Vergütung im Zusammenhang mit der Vermittlung von Versicherungsverträgen gewährt werden. Ähnliche Regelungen finden sich auch für die Schweiz, insbesondere im FINMA Rundschreiben 2010/1, welches unter anderem ein einfaches, transparentes und langfristig ausgerichtetes Vergütungssystem vorschreibt. Momentan entscheidet noch der Verwaltungsrat einer Genossenschaft als auch jener einer AG über seine eigene Vergütung. Ab März 2014 wenn die „Initiative gegen die Abzockerei“ rechtlich gültig ist verändert sich für börsenkotierte AGs die Situation jedoch: anstatt des Verwaltungsrats wird die Generalversammlung über die Höhe der Vergütung entscheiden.

Grigoleit 2013, S. 5-6).

${ }^{17}$ Für nicht börsennotierte Aktiengesellschaften und andere Rechtsformen ist die Anwendung der Regelungen lediglich empfohlen. Börsenkotierte Aktiengesellschaften müssen hingegen Abweichungen vom Kodex nach dem Comply or Explain Prinzip erklären. 
Es lässt sich festhalten, dass im Allgemeinen die Anforderungen an die Aufsichtsgremien von Versicherungen über alle Rechtsformen hinweg ähnlich sind. Ausnahmen bilden in Deutschland die KdöR und der kleinere Versicherungsverein. Die SE bietet zudem mehr Flexibilität bzgl. der Organisationsstruktur und der Arbeitnehmermitbestimmung. In der Schweiz hingegen lässt sich in den Anforderungen der Verwaltungsräte erkennen, dass sich die Zielvorgaben einer AG von einer Genossenschaft unterscheiden. Ab März 2014 werden sich zudem erheblich die Anforderungen an die Vergütungsverfahren nach Rechtsform unterscheiden.

\section{Ableitung von Gemeinsamkeiten und Unterschieden sowie Abgleich mit wissenschaftlichen Resultaten}

In Tab. 7 werden wesentliche Gemeinsamkeiten und Unterschiede im Vergleich vom schweizerischen und deutschen System zusammengetragen. Bzgl. der Organisation unterscheiden sich der Aufsichts- und Verwaltungsrat aufgrund der Umsetzung der Volksinitiative ,gegen die Abzockerei“ ab März 2014 in der Dauer der maximalen Wahlperiode. In Deutschland kann ein Aufsichtsrat maximal über fünf Jahre hinweg gewählt werden, in der Schweiz müssen demnächst die Wahlen für den Verwaltungsrat jährlich stattfinden. Ein weiterer Unterschied ergibt sich dadurch, dass der Aufsichtsrat paritätisch von Arbeitnehmer- und Eigenkapitalvertretern besetzt ist, im Verwaltungsrat jedoch ausschließlich die Eigenkapitalseite vertreten ist.

Die Verantwortlichkeiten des schweizerischen Verwaltungsrats gehen über die des deutschen Aufsichtsrats hinaus, da der Verwaltungsrat neben der Überwachungsfunktion auch eine Leitungsfunktion innehat. Die Aufgaben des Verwaltungsrats sind folglich umfangreicher als die des Aufsichtsrats. Der Swiss Code of Best Practice ist jedoch weniger bindend als der deutsche Corperate Governance Kodex, da nur letzterer ein im Gesetz verankertes Comply or Explain Prinzip enthält.

In der Festlegung der Vergütung von Aufsichtsrat und Verwaltungsrat beseitigt die „Abzocker Initiative“ Unterschiede. Bisher bestimmen Verwaltungsräte ihre Vergütung autonom ohne die Zustimmung der Aktionäre einholen zu müssen. Ab März 2014 gleichen sich hier jedoch die beiden Systeme an, da sowohl für den Aufsichtsrat als auch für den Verwaltungsrat die Entlohnung durch die Aktionäre beschlossen wird.

Auch im Bereich der speziellen Anforderungen aus dem Versicherungsbereich überwiegen die Gemeinsamkeiten. So finden sich in den deutschen sowie Schweizer Aufsichtsgesetzen „Fit-and-Proper“ Kriterien und die Aufsichtsbehörden haben die Befugnis Aufsichts- bzw. Verwaltungsräte abzuberufen, falls diese Kriterien nicht eingehalten werden. Nach dem Obligationenrecht wäre es möglich die Leitung der Geschäftsführung und des Verwaltungsrats einer Person zu übertragen, doch nach dem Versicherungsaufsichtsgesetz ist dies nur in Ausnahmefällen möglich und somit ist diese Dualität in der Versicherungswirtschaft sowohl in Deutschland als auch in der Schweiz ähnlich stark reguliert.

Auch ähneln sich die beiden Verordnungen (VersVergV und FINMA Rundschreiben 2010/1) zu Vergütungssystemen. Variable Bestandteile des Gehalts sollen sich am langfristigen wirtschaftlichen Erfolg des Unternehmens ausrichten und erst ver- 
Tab. 7 Ableitung von Gemeinsamkeiten und Unterschieden

\begin{tabular}{|c|c|c|}
\hline Kriterium & Gemeinsamkeiten & Unterschiede \\
\hline \multirow[t]{4}{*}{$\overline{\text { Organisation }}$} & $\begin{array}{l}\text { Wahlperiode in etwa gleich (max. } 5 \\
\text { oder } 6 \text { Jahre) (bisher) }\end{array}$ & $\begin{array}{l}\text { Wahlperiode unterschiedlich (max. } 5 \text { Jahre } \\
\text { in Deutschland oder } 1 \text { Jahr in der Schweiz) } \\
\text { (ab März 2014) }\end{array}$ \\
\hline & $\begin{array}{l}\text { Genehmigung durch Ge- } \\
\text { neralversammlung/ } \\
\text { Hauptversammlung }\end{array}$ & Keine Arbeitnehmer im VR \\
\hline & \multirow[t]{2}{*}{ Bildung von Ausschüssen } & $\begin{array}{l}\text { Mitgliederanzahl: mind. } 1 \text { (VR), mind. } 3 \\
\text { (AR) }\end{array}$ \\
\hline & & $\begin{array}{l}\text { Comply or Explain Prinzip bzgl. DCGK } \\
\text { (AR); SCBP bloße Empfehlung (VR) }\end{array}$ \\
\hline \multirow[t]{2}{*}{$\begin{array}{l}\text { Befugnisse und } \\
\text { Pflichten }\end{array}$} & $\begin{array}{l}\text { Mehrheitsentscheidung bei Beschluss- } \\
\text { fassungen; Entscheid beim Vorsitzen- } \\
\text { den bei Stimmengleichheit }\end{array}$ & $\begin{array}{l}\text { Monistisches (Schweiz) vs. dualistisches } \\
\text { System (Deutschland) }\end{array}$ \\
\hline & Haftungsregeln ungefähr gleich & $\begin{array}{l}\text { Kontrolle (AR) vs. Oberleitung und Kon- } \\
\text { trolle (VR) }\end{array}$ \\
\hline \multirow[t]{4}{*}{$\begin{array}{l}\text { Qualifikation } \\
\text { und Entlohnung }\end{array}$} & $\begin{array}{l}\text { Hauptversammlung/Generalversamm- } \\
\text { lung beschließt Entlohnung für AR/VR } \\
\text { (ab März 2014) }\end{array}$ & $\begin{array}{l}\text { Generalversammlung beschließt Ent- } \\
\text { lohnung für VR und Geschäftsleitung (ab } \\
\text { März 2014) }\end{array}$ \\
\hline & $\begin{array}{l}\text { AR/VR beschließt Entlohnung für Vor- } \\
\text { stand (bisher) }\end{array}$ & $\begin{array}{l}\text { AR bestimmt Entlohnung für Vorstand (ab } \\
\text { März 2014) }\end{array}$ \\
\hline & Offenlegung der Entlohnung & VR beschließt eigene Entlohnung (bisher) \\
\hline & & Max. 10 AR Mandate pro Person \\
\hline \multirow{4}{*}{$\begin{array}{l}\text { Spezielle An- } \\
\text { forderungen im } \\
\text { Versicherungs- } \\
\text { bereich }\end{array}$} & $\begin{array}{l}\text { Notwendiges Fachwissen und Erfah- } \\
\text { rung erforderlich (Fit-and-Proper) }\end{array}$ & $\begin{array}{l}\text { Anzahl früherer Geschäftsführer im AR } \\
\text { auf } 2 \text { beschränkt }\end{array}$ \\
\hline & $\begin{array}{l}\text { Abberufung von Aufsichts- bzw. Ver- } \\
\text { waltungsräten durch Aufsichtsbehörden } \\
\text { möglich }\end{array}$ & $\begin{array}{l}\text { Entlohnung AR darf nicht in Zusammen- } \\
\text { hang mit dem Verkauf von Versicherungs- } \\
\text { verträgen stehen }\end{array}$ \\
\hline & \multirow{2}{*}{$\begin{array}{l}\text { Dualität der Führung des Aufsichts- } \\
\text { organs und der Geschäftsleitung nicht } \\
\text { möglich }\end{array}$} & $\begin{array}{l}\text { VR für Vergütungsreglement für alle Mit- } \\
\text { arbeiter verantwortlich }\end{array}$ \\
\hline & & $\begin{array}{l}\text { AR nur für Vergütungssystem für Ge- } \\
\text { schäftsleitung verantwortlich }\end{array}$ \\
\hline
\end{tabular}

$A R$ deutscher Aufsichtsrat, $V R$ Schweizerischer Verwaltungsrat

zögernd in Anspruch genommen werden können. Der Verwaltungsrat hat jedoch bei dieser Thematik weitreichendere Befugnisse als der Aufsichtsrat. Ersterer erlässt das Vergütungssystem für das gesamte Unternehmen und nicht nur wie der Aufsichtsrat für den Vorstand bzw. die Geschäftsleitung. Ein weiterer Unterschied besteht darin, dass in Deutschland die Vergütung von Aufsichtsrats-Mitgliedern nicht im Zusammenhang mit dem Verkauf von Versicherungsverträgen stehen darf.

Tabelle 8 stellt zentrale Corporate Governance Faktoren in Bezug auf Aufsichtsgremien dar und nimmt einen Abgleich mit wissenschaftlichen Studien zu diesem Themenbereich vor. ${ }^{18}$ Wir bewerten anhand der Literatur den Einfluss verschiedener Eigenschaften des Aufsichtsgremiums auf die Performance und das Risiko. Unter

\footnotetext{
${ }^{18}$ Aufsichtsgremium, Aufsichtsorgan und Kontrollgremium gebrauchen wir als Synonyme für das oberste Kontrollorgan bei einem Versicherungsunternehmen. Je nach Aufsichtssystem kann dies der Aufsichtsrat (z. B. Deutschland), der Verwaltungsrat (z. B. Schweiz) oder das Board (z. B. UK oder USA) sein. Die meisten wissenschaftlichen Arbeiten beziehen sich auf Länder mit einer monistischen Unternehmensleitung.
} 


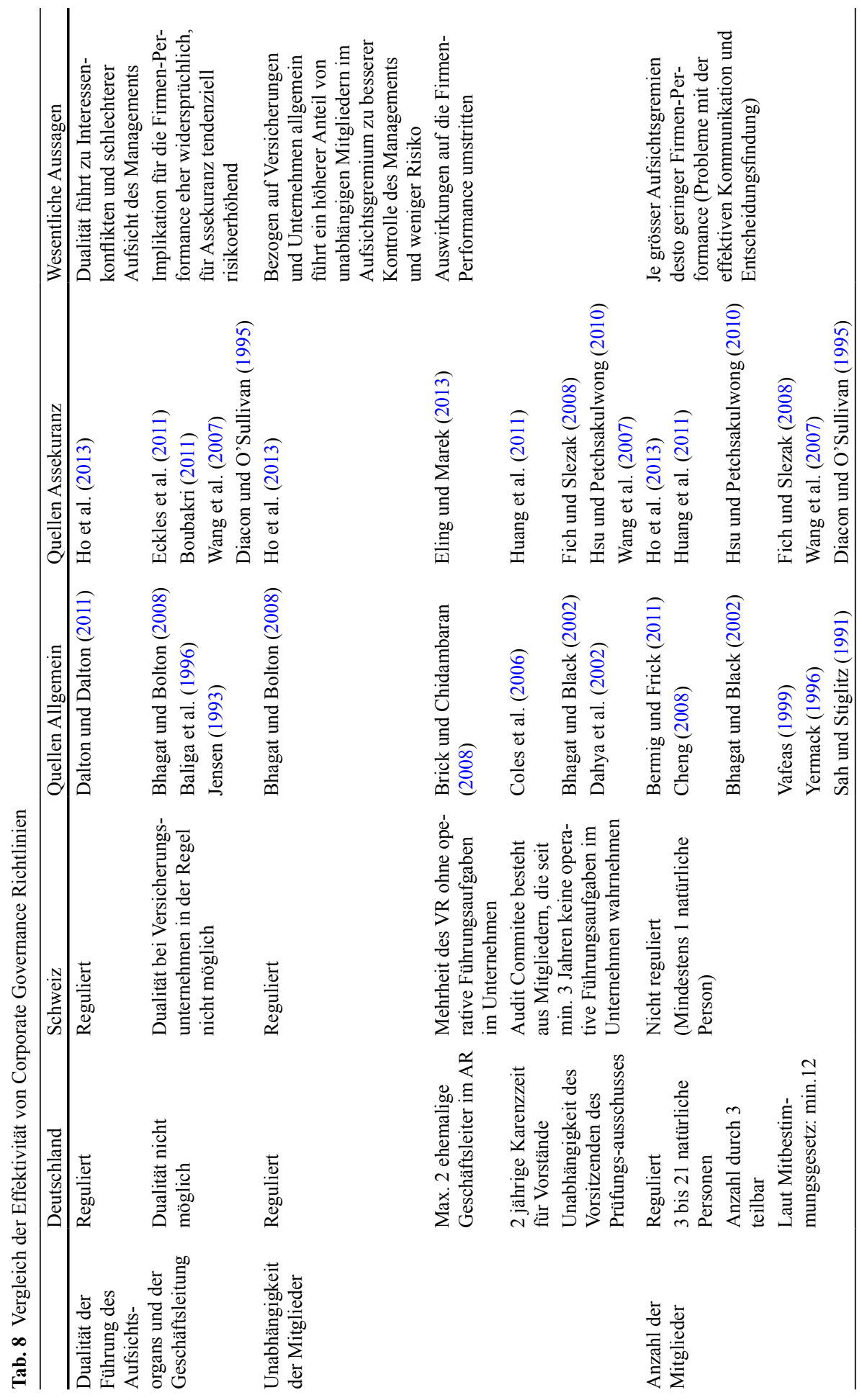



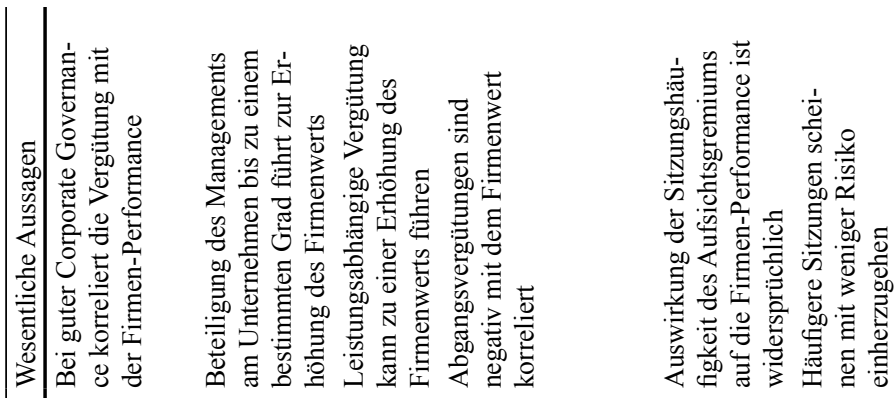

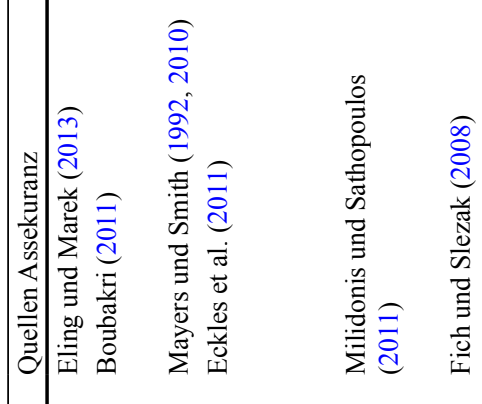

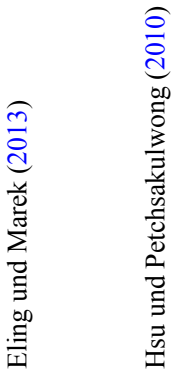

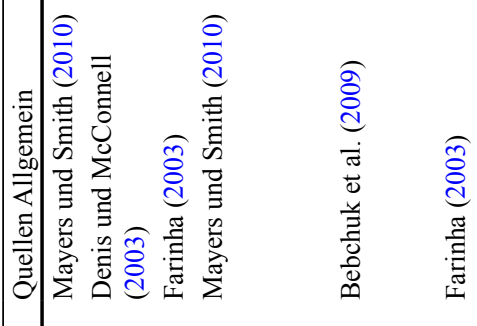

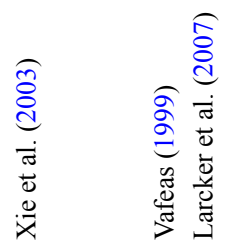

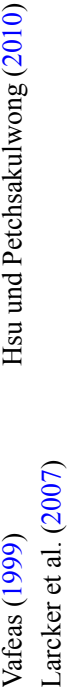

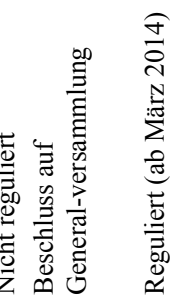

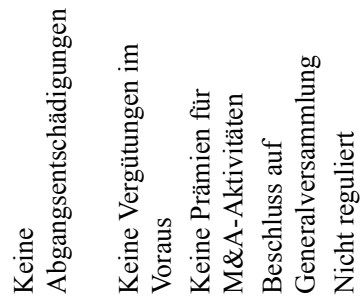

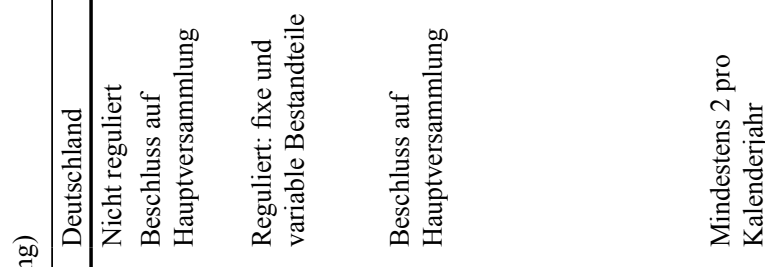

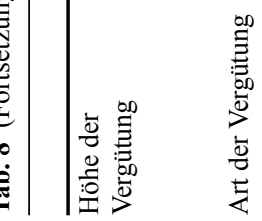

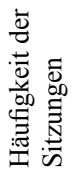




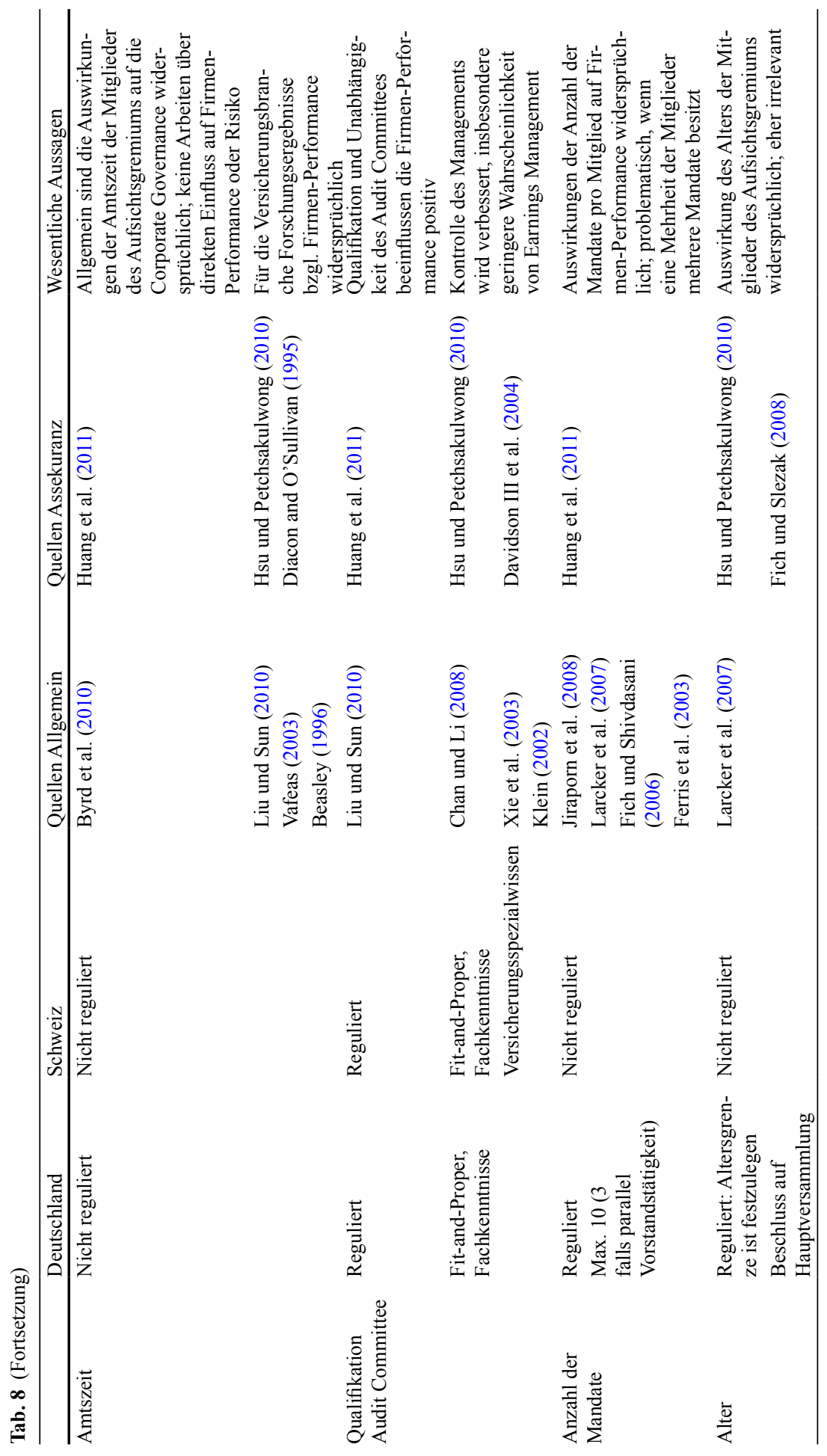


Performance wird der Erfolg eines Versicherungsunternehmens anhand verschiedener Kennzahlen gemessen, z. B. durch Profitabilität, Aktienkursentwicklung oder Kosteneffizienz. Die Risikoanalyse hingegen umfasst alle gängigen Risikotypen, wie Operational Risk, Underwriting Risk und Market Risk. In der Literatur wird das Risiko häufig gemessen durch die Standardabweichung des „Return on Assets“ (RoA) oder die Standardabweichung der „Loss Ratio“ (LR). ${ }^{19}$

Die am häufigsten untersuchten Eigenschaften von Aufsichtsgremien sind

a. die Dualität der Führung des Aufsichtsorgans und der Geschäftsleitung,

b. der Anteil der unabhängigen Mitglieder im Aufsichtsorgan,

c. die Anzahl der Mitglieder im Aufsichtsorgan und

d. die Vergütung der Mandatsträger.

Weitere Faktoren, die in der Literatur behandelt werden sind, die Art der Vergütung, die Häufigkeit der Sitzungen des Aufsichtsgremiums, die gesamte Amtszeit der einzelnen Mitglieder (mögliche Verweildauer im Aufsichtsgremium über alle Wahlperioden hinweg), die Qualifikation der Mitglieder des Audit Committees, die Anzahl der Mandate sowie das Alter der Mandatsträger.

Ad a) Dualität: Ein Vergleich bei der Regulierung zeigt folgende Gemeinsamkeiten und Unterschiede: Eine Dualität der Führung des Aufsichtsorgans und der Geschäftsleitung ist praktisch in beiden Systemen bei Versicherungen nicht möglich. Der Verwaltungsratspräsident dürfte prinzipiell zwar zugleich Vorsitzender der Geschäftsleitung sein, allerdings ist dies im Versicherungsbereich nicht gestattet (Art. 13 AVO, Ausnahmen möglich). Dies kann damit legitimiert werden, dass wenn die Führung der Geschäftsleitung und des Kontrollgremiums auf eine Person vereinigt wird, das Risiko des Versicherungsunternehmens tendenziell höher ist.

Ad b) Anteil der unabhängigen Mitglieder: Auch wird in Deutschland sowie in der Schweiz darauf hingewirkt, dass ein beträchtlicher Anteil der Mitglieder des Aufsichtsgremiums unabhängig ist. Hier sind die deutschen Richtlinien jedoch um einiges strenger, da die Vorgaben zum Teil aus dem Gesetz stammen und zum Teil aus dem Corporate Governance Kodex. Die Schweizer Vorgaben hingegen basieren hauptsächlich auf dem Swiss Code of Best Practice, welcher weniger maßgebend ist als der deutsche Kodex. Ob ein hoher Anteil von unabhängigen Mitgliedern im Aufsichtsorgan jedoch wünschenswert ist, ist in der Literatur umstritten. Zwar ist erwiesen, dass das Risiko des Versicherers sinkt, doch kann dies auch einhergehen mit sinkender Performance.

Ad c) Anzahl der Mitglieder: Die meisten Studien für Unternehmen allgemein sowie für Versicherer legen nahe, dass eine höhere Anzahl von Mitgliedern im Aufsichtsgremium sich vermindernd auf die Performance des Unternehmens auswirkt. Die am häufigsten gebrauchte Erklärung ist, dass große Aufsichtsgremien schwer zu koordinieren sind und lange zur Entscheidungsfindung brauchen. Ein weiteres Argument wird von Boubakri (2011) aufgrund von Sah und Stiglitz (1991) eingebracht: Mit steigender Anzahl von Mitgliedern im Aufsichtsgremium seien diese schwieriger

\footnotetext{
${ }^{19}$ Die Strukturen des Aufsichtsgremiums sowie die Eigenschaften der Mandatsträger wirken sich zudem auf die Güte des Reportings eines Unternehmens aus, wie z. B. bezüglich des Earnings Management (Vgl. Xie et al. 2003, Eckles et al. 2011 und Liu und Sun 2010).
} 
von riskanten, aber profitablen Projekten zu überzeugen. Die Aussage, dass ein großes Aufsichtsgremium zu geringerer Firmen-Performance führt wird von Bermig und Frick (2011) (Schweizer Daten) und Beiner et al. (2004) (deutsche Daten) empirisch überprüft und bezweifelt. Beide Studien geben jedoch an, dass sie eine Minderheitsmeinung vertreten.

Die Anzahl der Mitglieder wird in Deutschland im Gegensatz zu der Schweiz scharf reguliert. Die Aufsichtsratsgröße ist auf maximal 21 Personen begrenzt. Bei Unternehmen, die dem Mitbestimmungsgesetz unterliegen, ist die minimale Anzahl zwölf Personen (ansonsten drei). Die durchschnittliche Größe von Aufsichtsräten von Firmen welche dem Mitbestimmungsgesetz unterliegen betrug 2004 (1979) im Durchschnitt 15,2 (14,0) Personen (Vgl. Gerum und Debus 2006). Die tatsächliche Größe der Verwaltungsräte von im SMI kotierten Unternehmen betrug hingegen im Jahr 2007 im Durchschnitt 10,1 Personen (Vgl. Volonte 2011). Im Vergleich zu den späten 1980er/frühen 1990er Jahren ist die Größe des Verwaltungsrates ungefähr um vier Personen zurückgegangen. ${ }^{20}$

Ad d) Vergütung der Mandatsträger: Bei der Festlegung der Entlohnung der Mitglieder des Aufsichtsrats bzw. des Verwaltungsrats ergeben sich ab März 2014 kaum noch Unterschiede zwischen Deutschland und der Schweiz aufgrund der Annahme der „Volksinitiative gegen die Abzockerei“ in der Schweiz. In beiden Systemen wird die Vergütung durch die Aktionäre festgelegt.

Bei den restlichen Kriterien verhält es sich ähnlich - die deutschen und Schweizer Richtlinien gehen in dieselbe Richtung, nur die deutschen haben einen verbindlicheren Charakter. Eine Ausnahme bildet die Art der Vergütung. Hier sind die Schweizer Richtlinien um einiges strenger und verbieten aufgrund der oben genannten Volksinitiative verschiedene Vergütungsformen ab März 2014. Gegen Abgangsvergütungen lassen sich empirische Argumente in der Literatur finden. Z. B. sind diese mit einem niedrigen Firmenwert korreliert. Die kausalen Zusammenhänge sind jedoch nicht klar (Vgl. Bebchuk 2009) und so scheint es fraglich ob ein Verbot dieser Vergütungskomponente eine geeignete Maßnahme ist.

Die Anforderungen an das Audit Committee und deren Mitglieder sind in beiden Ländern streng reguliert und bei Versicherungen wird gesondertes Spezialwissen vorausgesetzt. Dies kann damit legitimiert werden, dass ein unabhängiges und qualifiziertes Audit Committee sich nachweislich positiv auf die Firmen-Performance und die Corporate Governance auswirkt. Die Häufigkeit der Sitzungen des Aufsichts- bzw. des Verwaltungsrats sind in Deutschland kaum (zwei Sitzungen pro Kalenderjahr) und in der Schweiz nicht reguliert. Die Amtszeit eines Mitglieds des Aufsichtsgremiums ist weder in Deutschland noch in der Schweiz reguliert. Die nicht bzw. kaum vorhandene Regulierung bei beiden Faktoren kann als effektiv angesehen werden, da wissenschaftliche Erkenntnisse hierzu nicht eindeutig sind.

Die Anzahl der Mandate pro Mitglied ist in Deutschland im Gegensatz zu der Schweiz reguliert. In der Literatur sind negative Konsequenzen für die FirmenPerformance erst nachweisbar, wenn eine Mehrheit der Mitglieder des Aufsichts-

\footnotetext{
${ }^{20}$ Siehe auch Beiner et al. (2004). Sie betrachten ein Sample von 165 Schweizer Firmen welche SWX kotiert sind (ohne Banken und Versicherungen). Die Verwaltungsratsgröße beträgt im Durchschnitt sechs Personen.
} 
gremiums weitere Mandate besitzen. Einerseits wird die Hypothese vertreten, dass Mandatsträger mit vielen Aufsichtsmandaten nicht genug Zeit für die Erfüllung ihrer Aufgaben besitzen. Andererseits wird argumentiert, dass mehrere Mandate die Expertise des einzelnen Mitglieds erhöhen. Aus diesem Grunde ist fraglich, ob eine Regulierung per Gesetz auf Ebene des einzelnen Mitglieds des Aufsichtsgremiums effektiv ist. In Deutschland im Gegensatz zur Schweiz muss eine Altersgrenze auf der Hauptversammlung für Mitglieder des Aufsichtsgremiums festgelegt werden. Wissenschaftliche Studien zeigen jedoch, dass dieser Faktor eher nicht signifikant für die Firmen-Performance oder das Risiko ist.

Folgende Faktoren haben signifikanten Einfluss auf die Performance sowie die Risikoausrichtung des Unternehmens und sind in Deutschland und der Schweiz ähnlich reguliert: Dualität der Führung der Geschäftsleitung und des Aufsichtsgremiums, Unabhängigkeit der Mitglieder, Anzahl der Mitglieder, Höhe und Art der Vergütung und Qualifikation der Mitglieder des Audit Committees. Die Faktoren Häufigkeit der Sitzungen und Amtszeit, Anzahl der Mandate sowie Alter eines Mitglieds des Aufsichtsgremiums besitzen keinen signifikanten Einfluss, bzw. ihre Auswirkungen auf die Performance und die Risikoausrichtung des Unternehmsnes sind noch nicht bekannt. Nichtsdestotrotz sind diese Faktoren in Deutschland reguliert.

Es lässt sich zusammenfassen, dass die deutschen Governance Richtlinien bzgl. des Aufsichtsrats strenger zu sein scheinen als die Schweizer Richtlinien bzgl. des Verwaltungsrats. Jedoch ist die Regulierung in der Schweiz tendenziell passgenauer und ist ausschließlich auf die wesentlichen Faktoren fokussiert, so dass die Schweizer Regulierung effektiver erscheint. ${ }^{21}$

\section{Fazit und Entwicklungstendenzen}

Der grundlegende Unterschied zwischen dem deutschen Aufsichtsrat und dem Schweizer Verwaltungsrat liegt in der Tatsache, dass der Aufsichtsrat ein reines Kontrollgremium ist und der Verwaltungsrat neben der Kontrollfunktion zudem die Oberleitung des Unternehmens verantwortet. Ein weiterer wichtiger Unterschied ist die paritätische Besetzung des Aufsichtsrats, welche in der Schweiz nicht stattfindet. Neben diesen beiden Punkten ist jedoch eine zunehmende Tendenz zu einer Annäherung der beiden Strukturtypen des Schweizer und deutschen Systems festzustellen. Diese Konvergenz ist darauf zurückzuführen, dass beide Systeme Nachteile haben und diese im Laufe der Zeit immer offensichtlicher geworden sind. Man sucht letztlich den Ausgleich für Schwächen des einen Systems in gezielten Anleihen beim anderen (Vgl. Böckli 2008). Ein aktuelles Beispiel ist die Schweizer Volksinitiative „gegen die Abzockerei“, wodurch die Schweiz nicht nur bei der Regulierung der Entlohnung von Verwaltungsräten und der Geschäftsführung im Vergleich zu Deutsch-

\footnotetext{
${ }^{21}$ Bei der Bewertung der Effektivität von Corporate Governance Richtlinien sollte beachten werden, dass die Auswirkungen der Strukturen und Eigenschaften des Aufsichtsgremiums je nach Rechtsform unterschiedlich ausfallen können. Es gibt Unterschiede bei den vorhandenen Interessenkonflikten und den externen Kontrollmechanismen, etwa durch Analysten, wobei unterschiedliche Resultate in der Literatur bestehen (Vgl. Piotroski und Roulstone 2004, Cole et al. 2011, Cummins und Nini 2002 und Doherty und Dionne 1993).
} 
land aufgeholt hat, sondern nun die strengeren Richtlinien in diesem Bereich besitzt. Seit der Einführung der Societas Europaea ist es auch in Deutschland theoretisch möglich für einen Versicherer sich für das monistische System zu entscheiden, auch wenn dies in der Versicherungspraxis bislang noch nicht anzutreffen ist.

Ein Rechtsformvergleich der verschiedenen Anforderungen an die Aufsichtsgremien der Versicherer zeigt, dass im Allgemeinen die Regelungen für Versicherungen über alle Rechtsformen hinweg ähnlich sind. In Deutschland sind die Anforderungen an AGs, SEs und VVaGs ähnlich ausgeprägt. Die SE besitzt jedoch mehr Flexibilität bei der Frage der Arbeitnehmermitbestimmung und der Organisation des Aufsichtsgremiums. Kleinere VVaGs sowie KdöR sind jedoch von einer Reihe von Anforderungen ausgenommen. Auch in der Schweiz ähneln sich die Rechtsform der Genossenschaft und der AG. Ein Unterschied besteht jedoch darin, dass der Verwaltungsrat der AG die Gewinnorientierung der Gesellschaft berücksichtigen muss und jener der Genossenschaft die Förderung der genossenschaftlichen Aufgabe. Des Weiteren gilt die „Abzocker Initiative“ nur für börsenkotierte AGs und somit werden die Auflagen für das Vergütungsverfahren für AGs ab März 2014 weit strenger ausfallen als für Genossenschaften.

Bei der generellen Frage nach der Effektivität lässt sich ein leichter Vorsprung der Schweizer Corporate Governance Richtlinien ausmachen. Zwar decken die Regulierungen in beiden Ländern die wichtigsten Aspekte der Corporate Governance ab, aber in Deutschland werden zudem Bereiche reguliert, die empirisch keinen signifikanten Einfluss auf die Performance oder Risikoausrichtung des Versicherungsunternehmens besitzen. Somit schießt Deutschland in einigen Punkten über das Ziel hinaus. Des Weiteren haben die deutschen Corporate Governance Richtlinien im Allgemeinen einen verbindlicheren Charakter als ihr Schweizer Pendant.

In Zukunft ist damit zu rechnen, dass die Corporate Governance Regeln in Deutschland und der Schweiz zunehmend verstärkt werden. So hat am 16. April 2013 das Europäische Parlament einem Gesetzentwurf der EU-Kommission zugestimmt, der die Bonuszahlungen an Bankmitarbeiter begrenzt und EU-Binnenmarktkommissar Barnier hat angekündigt, dass die EU-Kommission bis Jahresende einen Gesetzesvorschlag vorlegen wird zur allgemeinen Begrenzung von Managergehältern. In der Schweiz geht der Trend in die gleiche Richtung und so wird demnächst über die Initiative ,1:12- Für gerechte Löhne“ abgestimmt, welche festlegen würde, dass das höchste Gehalt in einem Unternehmen maximal dem zwölffachen des tiefsten Gehalts entsprechen darf. Der verstärkte Regulierungsdruck bezieht sich jedoch nicht nur auf Fragen der Entlohnung des Top-Managements. In Deutschland zeichnet sich zunehmend die Einführung einer Frauenquote für Aufsichtsräte ab. Am 18. April 2013 wurde eine Gesetzesinitiative im deutschen Bundestag hierzu zwar abgelehnt, aber EU-Justizkommissarin Reding versucht weiterhin auf EU-Ebene eine Frauenquote von $40 \%$ für Aufsichtsräte bis 2020 einzuführen. ${ }^{22}$ Schlussendlich ist davon auszugehen, dass Aufsichts- und Verwaltungsräte in Zukunft stärker reguliert werden. Die Frage ist nur, ob ein engeres Corporate Governance Korsett den verschiedenen Stakeholdern wirklich Vorteile bringt.

\footnotetext{
${ }^{22}$ Vgl. hierzu auch den Aktionsplan: Europäisches Gesellschaftsrecht und Corporate Governance der Europäische Kommission (2012).
} 


\section{Appendix: Abkürzungen}

\begin{tabular}{|c|c|}
\hline $\mathrm{AG}$ & Aktiengesellschaft \\
\hline AR & Aufsichtsrat \\
\hline AktG & Aktiengesetz \\
\hline AVO & Aufsichtsverordnung \\
\hline BerVersV & Versicherungsberichterstattungs-Verordnung \\
\hline BilReG & Bilanzrechtsreformgesetz \\
\hline BilMoG & Bilanzrechtsmodernisierungsgesetz \\
\hline BV & Bundesverfassung \\
\hline DCGK & Deutscher Corporate Governance Kodex \\
\hline GenG & Genossenschaftsgesetz \\
\hline KdöR & Körperschaft des öffentlichen Rechts \\
\hline KonTraG & Gesetz zur Kontrolle und Transparenz im Unternehmensbereich \\
\hline OR & Obligationenrecht \\
\hline RechVersV & Versicherungsunternehmens-Rechnungslegungsverordnung \\
\hline RLCG & SIX Swiss Exchange Richtlinie Corporate Governance \\
\hline SCBP & Swiss Codex of Best Practice \\
\hline SE & Societas Europaea \\
\hline SEAG & SE-Ausführungsgesetz \\
\hline SEBG & SE-Beteiligungsgesetz \\
\hline SE-VO & SE-Verordnung \\
\hline TrasPuG & Transparenz- und Publizitäts-Gesetz \\
\hline UMAG & $\begin{array}{l}\text { Gesetz zur Unternehmensintegrität und Modernisierung des } \\
\text { Anfechtungsrechts }\end{array}$ \\
\hline VAG & Versicherungsaufsichtsgesetz \\
\hline VersVergV & Versicherungsvergütungsverordnung \\
\hline VorstAG & Gesetz zur Angemessenheit der Vorstandsvergütung \\
\hline VorstOG & Vorstandsvergütungs-Offenlegungsgesetz \\
\hline VVaG & Versicherungsverein auf Gegenseitigkeit \\
\hline VR & Verwaltungsrat \\
\hline
\end{tabular}

\section{Literatur}

Baliga, B.R., Moyer, R.C, Rao, R.S.: CEO duality and firm performance: what's the fuss? Strategic Manag. J. 17, 41-43 (1996)

Bauen, M., Venturi, S.: Der Verwaltungsrat. Schulthess, Zürich (2007)

Bähr, G.W., Bürkle, J., Göertz, S., Kaulbach, D., Pohlmann, P.: Versicherungsaufsichtsgesetz - VAG-. Beck, München (2012)

Beasley, M.S.: An empirical analysis of the relation between the board of director composition and financial statement fraud. Account. Rev. 71(4), 443-465 (1996)

Bebchuk, L., Cohen, A., Ferrell, A.: What matters in Corporate Governance? Rev. Financial Stud. 22(2), 783-827 (2009)

Beiner, S., Drobetz, W., Schmid, F., Zimmermann, H.: Is board size an independent corporate governance mechanism? Kyklos 57(3), 327-356 (2004)

Berliner Initiativkreis: German Corporate Governance Codex. Berlin (2002) 
Bermig, A., Frick, B.: Determinanten der „Übergröße“ deutscher Aufsichtsräte. Schmollers Jahrb. 131(1), 169-194 (2011)

Bhagat, S., Black, B.: The non-correlation between board independence and long-term firm performance. J. Corp. Law 27(2), 231-273 (2002)

Bhagat, S., Bolton, B.: Corporate Governance and firm performance. J. Corp. Finance 14(3), 257-273 (2008)

Boubakri, N.: Corporate governance and issues from the insurance industry. J. Risk Insur. 78(3), 501-518 (2011)

Böckli, P.: Konvergenz: Annäherung des monistischen und des dualistischen Führungs- und Aufsichtssystems. In: Hommelhoff, O., Hopt, K.J., v. Werder, A. (Hrsg.) Handbuch Corporate Governance. Schäffer-Poeschel, Stuttgart (2008)

Brick, I.E., Chidambaran, N.K.: Board monitoring, firm risk, and external regulation. J. Regul. Econ. 33, $87-116(2008)$

Byrd, J., Cooperman, E.S., Wolfe, G.A.: Director tenure and the compensation of bank CEOs. Manag. Finance 36(2), 86-102 (2010)

Chan, K.C., Li, J.: Audit committee and firm value: evidence on outside top executives as expert-independent directors. Corp. Gov.: Int. Rev. 16(1), 16-31 (2008)

Cheng, S.: Board size and the variability of corporate performance. J. Financial Econ. 87, 157-176 (2008)

Cole, C.R., He, E., McCullough, K.A., Semykina, A., Sommer, D.W.: An empirical examination of stakeholder groups as monitoring sources in Corporate Governance. J. Risk Insur. 78(3), 703-730 (2011)

Coles, J.L., Daniel, N.D., Naveen, L.: Managerial incentives and risk-taking. J. Financial Econ. 79, 431486 (2006)

Cummins, J.D., Nini, G.P.: Optimal capital utilization by financial firms: evidence from the property-liability insurance industry. J. Finaical Serv. Res. 21(1/2), 15-53 (2002)

Dahya, J., McConnell, J.J., Travlos, N.G.: The cadbury committee, corporate performance, and top management turnover. 57(1), 461-483 (2002)

Dalton, D.R., Dalton C.M.: Integration of micro and macro studies in governance research: CEO duality, board composition, and financial performance. J. Manag. 37(2), 404-411 (2011)

Davidson III, W.N., Xie, B., Xu, W.: Market reaction to voluntary announcements of audit committee appointments: the effect of financial expertise. J. Account. Public Policy 23(4), 279-293 (2004)

Denis, K., McConnel, J.J.: International Corporate Governance. J. Financial Quant. Anal. 38(1) (2003)

Diacon, S.R., O'Sullivan, N.: Does Corporate Governance influence performance? Some evidence from U.K. insurance companies. International Rev. Law Econ. 15(4), 405-424 (1995)

Doherty, N., Dionne, G.: Insurance with undiversified risk: contract structure and organizational form of insurance firms. J. Risk Uncertain. 6(2), 187-203 (1993)

Downs, D.H., Sommer, D.W.: Monitoring, ownership, and risk-taking: the impact of guaranty funds. J. Risk Insur. 66(3), 477-497 (1999)

Eckles, D.L., Halek, M., He, E., Sommer, D.W., Zhang R.: Earnings smoothing, executive compensation, and Corporate Governance: evidence from the property-liability insurance industry. J. Risk Insur. 78(3), 761-790 (2011)

Eling, M., Marek, S.D.: Coporate Governance and risk taking: evidence from the U.K. and German insurance Markets. J. Risk Insur. (2013) doi:10.1111/j.1539-6975.2012.01510.x

Europäische Kommission: Aktionsplan: Europäisches Gesellschaftsrecht und Corporate Governance - ein moderner Rechtsrahmen für engagiertere Aktionäre und besser überlebensfähige Unternehmen. Com (2012) 740 final (2012)

Fahrina, J.: Corporate Governance: a survey of the literature. Working paper, Universidade do Porto (2003)

Ferris, S.P., Jagannathan, M., Pritchard, A.C.: Too busy to mind the business? Monitoring by directors with multiple board appointments. J. Finance 68(3), 1087-1111 (2003)

Fich, E.M., Shivdasani A.: Are busy boards effective monitors? J. Finance 71(2), 689-724 (2006)

Fich, E.M., Slezak, S.L.: Can Corporate Governance save distressed firms from bankruptcy? An empirical analysis. Geneva Pap. 30(2), 225-251 (2008)

Forstmoser, P.: Monistische oder dualistische Unternehmensverfassung? Das Schweizer Konzept. Working Paper, Universität Zürich (2003)

Gerum, E., Debus, M.: Die Größe des Aufsichtsrats als rechtspolitisches Problem - Einige empirische Befunde. Studie, Philipps-Universität Marburg (2006)

Grigoleit, H.C. (Hrsg.): Aktiengesetz Kommentar. Beck, München (2013) 
Grundei, J., Zaumseil, P.: Der Aufsichtsrat in der Corporate Govemance-Diskussion. In: Grundei, J., Zaumseil, P. (Hrsg.) Der Aufsichtsrat im System der Corporate Governance, S. 15-25. Gabler Verlag, Wiesbaden (2012)

Grün, A., Schmitz, J.: Personelle Besetzung des Vorstandes. In: Grundei, J., Zaumseil, P. (Hrsg.) Der Aufsichtsrat im System der Corporate Governance, S. 95-111 (2012)

Ho, C.-L., Lai, G.C., Lee, J.-P.: Organizational structure, board composition, and risk taking in the U.S. property casualty insurance industry. J. Risk Insur. 80(1), 169-203 (2013)

Honsell, H., Vogt, N.P., Watter, R. (Hrsg.): Basler Kommentar Obligationenrecht II. Helbling Lichtenhahn Verlag, Basel (2008)

Hönsch, H., Kaspar, M.: Vergütung der Aufsichtsratsmitglieder. In: Grundei, J., Zaumseil, P. (Hrsg.) Der Aufsichtsrat im System der Corporate Governance, S. 256-257 (2012)

Hsu, W.-Y., Petchsakulwong, P.: The impact of Corporate Governance on the efficiency performance of the Thai non-life insurance industry. Geneva Pap. 35, 28-49 (2010)

Huang, L., Lai, G.C., McNamara, M., Wang, J.L.: Corporate Governance and efficiency: evidence from U.S. property-liability insurance industry. J. Risk Insur. 78(3), 519-550 (2011)

Jensen, M.C.: The modern industrial revolution, exit, and the failure of internal control systems. J. Finance 48(3), 831-880 (1993)

Jiraporn, P., Kim, Y.S., Davidson III, W.N.: Multiple directorships and corporate diversification. J. Empir. Finance 15(3), 418-435 (2008)

Klein, A.: Audit committee, board of director characteristics, and earnings management. J. Account. Econ. 33(3), 375-400 (2002)

Larcker, D.F., Richardson, S.A., Tuna I.: Corporate Governance, accounting outcomes, and organizational performance. Account. Rev. 82(4), 963-1008 (2007)

Leube, B.: Personelle Besetzung des Aufsichtsrates: Qualifikationsanforderungen und Auswahl der Aufsichtsratsmitglieder. In: Grundei, J., Zaumseil, P. (Hrsg.) Der Aufsichtsrat im System der Corporate Governance, S. 202-219 (2012)

Liu, G., Sun, J.: Director tenure and independent audit committee effectiveness. Int. Res. J. Finance Econ. 51, 176-189 (2010)

Mayers, D., Shivdasani, A., Smith, C.W.: Board composition and corporate control: evidence from the insurance industry. J. Bus. 70(1), 33-62 (1997)

Mayers, D., Smith, C.W.: Executive compensation in the life insurance industry. J. Bus. 65(1), 51-74 (1992)

Mayers, D., Smith, C.W.: Compensation and board structure: evidence from the insurance industry. J. Risk Insur. 77(2), 297-327 (2010)

Milidonis, A., Stathopoulos, K.: Do U.S. insurance firms offer the „wrong“ incentives to their exectutives? J. Risk Insur. 78(3), 643-672 (2011)

Müller, R.: Entwicklung und Bedeutung der Corporate Governance. Working paper, Universität St. Gallen (2008)

Müller, R., Lipp, L., Plüss, A.: Der Verwaltungsrat. Schulthess, Zürich (2007)

Piotroski, J.D., Roulstone, D.T.: The influence of analysts, institutional investors, and insiders on the incorporation of market, industry, and firm-specific information into stock prices. Account. Rev. 79(4), 1119-1151 (2004)

Pohlmann, P.: Aufsichtsrechtliche Anforderungen an Schlüsselfunktionsträger in Versicherungsunternehmen. Working paper, Universität Münster (2012)

Prigge, S.: Überwachung der Vorstandstätigkeit. In: Grundei, J., Zaumseil, P. (Hrsg.) Der Aufsichtsrat im System der Corporate Governance, S. 77-92 (2012)

Roth, M.: Information und Organisation des Aufsichtsrats. Z. Unternehm. Ges.r. 41(2/3), 343-381 (2012)

Sah, R.K., Stiglitz, J.E. The quality of managers in centralized versus decentralized organizations. Q. J. Econ. 106(1), 289-295 (1991)

Vafeas, N.: Board meeting frequency and firm performance. J. Financial Econ. 53(1), 113-142 (1999)

Vafeas, N.: Length of board tenure and outside director independence. J. Bus. Finance Account. 30(7/8), 1043-1064 (2003)

Van Betteray, W., Heerma, P.H.: Haftung der Mitglieder des Aufsichtsrats in der Krise des Unternehmens. In: Dehnen, P.H. (Hrsg.) Der professionelle Aufsichtsrat, S. 51-75 (2011)

Volonte, C.: Wie hat sich die Struktur des Verwaltungsrates schweizerischer Firmen in den beiden letzten beiden Jahrzenten verändert? Working paper, Universität Basel (2011)

Welge, M.K., Eulerich, M.: Corporate-Governanee-Management. Gabler Verlag, Wiesbaden (2012) 
Werder, A.: Neue Entwiclungen der Corporate Governance in Deutschland. Schmalenbachs Z. f. betriebsw. Forsch. 63(1), 48-62 (2011)

Wang, J.L., Jeng V., Peng, J.L.: The impact of Corporate Governance structure on the efficiency performance of insurance companies in Taiwan. Geneva Pap. 32(2), 264-282 (2007)

Xie, B., Davidson III, W.N., DaDalt, P.J.: Earnings management and corporate governance: the role of the board and the audit committee. J. Corp. Finance 9(3), 295-316 (2003)

Yermack, D. Higher market valuation of companies with a small board of directors. J. Financial Econ. 40(2), 185-211 (1996)

Zaumseil, P.: Die Haftung des Aufsichtsrats auf Schadensersatz. In: Grundei, J., Zaumseil, P. (Hrsg.) Der Aufsichtsrat im System der Corporate Governance, S. 419-443 (2012)

Ziegler, U., Gey, A.: Arbeitnehmermitbestimmung im Aufsichtsrat der Europäischen Gesellschaft (SE) im Vergleich zum Mitbestimmungsgesetz. Betr.berat. 64(33), 1750-1758 (2009) 